\title{
MuSK Frizzled-Like Domain Is Critical for Mammalian Neuromuscular Junction Formation and Maintenance
}

\author{
Julien Messéant, ${ }^{1}$ Alexandre Dobbertin, ${ }^{1}$ Emmanuelle Girard, ${ }^{2}$ Perrine Delers, ${ }^{1}{ }^{\oplus}$ Marin Manuel, ${ }^{1}$ Francesca Mangione, ${ }^{3}$ \\ Alain Schmitt, ${ }^{4}$ Dominique Le Denmat, ${ }^{3}$ Jordi Molgó, ${ }^{5}$ Daniel Zytnicki, ${ }^{1}$ Laurent Schaeffer, ${ }^{2}$ Claire Legay, ${ }^{1}$ \\ and $\odot$ Laure Strochlic ${ }^{1}$ \\ ${ }^{1}$ Centre National de la Recherche Scientifique UMR 8119, Institut National de la Santé et de la Recherche Médicale U686, Université Paris Descartes, \\ Sorbonne Paris Cité, 75270 Paris cedex 06 France, ${ }^{2}$ Centre National de la Recherche Scientifique UMR 5239, Université Lyon 1, ENS, 69364 Lyon cedex 07 \\ France, ${ }^{3}$ EA 2496-PIPA, Université Paris Descartes, Sorbonne Paris Cité, 75270 Paris cedex 06 France, ${ }^{4}$ Centre National de la Recherche Scientifique Unité \\ Mixte de Recherche 8104, Institut National de la Santé et de la Recherche Médicale U1016, Institut Cochin, Université Paris Descartes, Sorbonne Paris Cité, \\ 75014 Paris, France, and ${ }^{5}$ Centre National de la Recherche Scientifique, Laboratoire de Neurobiologie et Développement, UPR 3294, Gif sur Yvette, Cedex \\ 91198, France
}

The muscle-specific kinase MuSK is one of the key molecules orchestrating neuromuscular junction (NMJ) formation. MuSK interacts with the Wnt morphogens, through its Frizzled-like domain (cysteine-rich domain [CRD]). Dysfunction of MuSK CRD in patients has been recently associated with the onset of myasthenia, common neuromuscular disorders mainly characterized by fatigable muscle weakness. However, the physiological role of Wnt-MuSK interaction in NMJ formation and function remains to be elucidated. Here, we demonstrate that the CRD deletion of MuSK in mice caused profound defects of both muscle prepatterning, the first step of NMJ formation, and synapse differentiation associated with a drastic deficit in AChR clusters and excessive growth of motor axons that bypass AChR clusters. Moreover, adult MuSK $\Delta C R D$ mice developed signs of congenital myasthenia, including severe NMJs dismantlement, muscle weakness, and fatigability. We also report, for the first time, the beneficial effects of lithium chloride, a reversible inhibitor of the glycogen synthase kinase-3, that rescued NMJ defects in MuSK $\Delta C R D$ mice and therefore constitutes a novel therapeutic reagent for the treatment of neuromuscular disorders linked to Wnt-MuSK signaling pathway deficiency. Together, our data reveal that MuSK CRD is critical for NMJ formation and plays an unsuspected role in NMJ maintenance in adulthood.

Key words: congenital myasthenic syndrome; lithium chloride; MuSK; neuromuscular junction; synaptogenesis; Wnt

\section{Introduction}

The formation of the neuromuscular junction (NMJ) relies upon the accurate apposition and contact between presynaptic nerve terminals and postsynaptic muscle targets. The muscle-specific tyrosine kinase receptor MuSK and its coreceptor Lrp4 (low density lipoprotein receptor) constitute the central hub orchestrating

Received Aug. 13, 2014; revised Feb. 6, 2015; accepted Feb. 16, 2015.

Author contributions: J. Messéant, A.D., E.G., P.D., M.M., F.M., A.S., and L. Strochlic performed research; J. Messéant, A.D., E.G., and L. Strochlic analyzed data; J. Messéant, A.D., E.G., C.L., and L. Strochlic wrote the paper; D.L.D., J. Molgó, D.Z., L. Schaeffer, C.L., and L. Strochlic designed research.

This work was supported by Association Française contre les Myopathies Grant 14960 to L. Strochlic and Grant 18046 to C.L., Centre National de la Recherche Scientifique, and Paris Descartes University. We thank Markus Rüegg for MuSK atibodies and technical advice; Sophie Nicole and Suzie Lefebvre for critical reading of the manuscript; Eric Krejci and Jean and Annie Cartaud for experimental advice; Anne-Sophie Armand and Capucine Trollet for help with muscle primary cultures; and Jean-Maurice Petit for technical advices and image processing. The mouse mutant line was established at the Mouse Clinical Institute (Institut Clinique de la Souris) in the Targeted Mutagenesis and Transgenesis Department with funds from the Institut National de la Santé et de la Recherche Médicale and the Association Française contre les Myopathies (Association Française contre les Myopathies Grant 14960 to L. Strochlic).

The authors declare no competing financial interests.

Correspondence should be addressed to Dr. Laure Strochlic, Centre National de la Recherche Scientifique Unité Mixte de Recherche 8119, Université Paris Descartes, PRES Sorbonne Paris Cité, Paris, France. E-mail: laure.strochlic@inserm.fr.

DOI:10.1523/JNEUROSCI.3381-14.2015

Copyright $\odot 2015$ the authors $\quad 0270-6474 / 15 / 354926-16 \$ 15.00 / 0$ all steps of NMJ formation and maintenance (Wu et al., 2010; Burden et al., 2013). MuSK contains in its extracellular region a Frizzled-like domain (cysteine-rich domain [CRD]) mediating its interaction with several Wnts molecules, including Wnt4, Wnt11, and Wnt9a in vitro (Jing et al., 2009; Strochlic et al., 2012; Zhang et al., 2012). Activation of the MuSK-Lrp4 complex regulates the prepatterning step, before muscle innervation, during which AChRs begin to aggregate in a broad central and prospective synaptic region of the muscle (Lin et al., 2001; Yang et al., 2001; Arber et al., 2002; Weatherbee et al., 2006; Kim and Burden, 2008). Moreover, in vivo knockdown of Wnt4 and Wnt11 affects muscle prepatterning and axon guidance, indicating a role for Wnt signaling in this process (Jing et al., 2009; Gordon et al., 2012; Strochlic et al., 2012). Upon innervation, the MuSK-Lrp4 complex is further stimulated by neural agrin, which induces multiple signaling pathways leading to clustering and remodeling of aneural AChR clusters (Kim et al., 2008; Zhang et al., 2008; Wu et al., 2010). In addition to their role in prepatterning, Wnt proteins have been shown to regulate agrin-induced AChR clustering in vitro (Henriquez et al., 2008; Wang et al., 2008). However, the general role of Wnt-induced mammalian NMJ formation and maintenance in adult life mediated by MuSK remains largely unknown. 
Mutations in genes or autoantibodies directed against proteins critical for NMJ formation or function are responsible for myasthenia, respectively congenital (CMS) or acquired (myasthenia gravis), mainly characterized by fatigable muscle weakness resulting from defective neuromuscular transmission (Hantaï et al., 2013; Berrih-Aknin et al., 2014). Recently, several cases of CMS linked to mutations in the CRD of MuSK as well as autoantibodies against the CRD and Ig1/2 domains of MuSK in antiAChR negative myasthenia gravis patients were identified, indicating that dysfunction of MuSK CRD is associated with the onset of myasthenia (Mihaylova et al., 2009; Takamori, 2012, 2013).

In the present study, to get insight into the functional role of Wnt-MuSK-induced NMJ formation and maintenance in vivo, we generated knock-out mice in which MuSK CRD $(M u S K \Delta C R D)$ was deleted. Analysis of the NMJ phenotype of $M u S K \triangle C R D$ embryos revealed profound defects in both muscle prepatterning and synapse differentiation. More surprisingly, we found that MuSK $\triangle C R D$ adult mice exhibited dislocation of NMJs and fatigable muscle weakness characteristic of CMS-like symptoms. We also demonstrated, for the first time, that lithium chloride ( $\mathrm{LiCl})$, a well-known reversible inhibitor of the glycogen synthase kinase-3 (Gsk3) rescued NMJ defects in MuSK $\Delta C R D$ embryos and adult mice.

Collectively, our data demonstrate that the absence of MuSK CRD affects NMJ formation from the prepatterning step to NMJ maintenance in adult, leading to a pathogenic phenotype. Importantly, the biological benefits of $\mathrm{LiCl}$ treatment on NMJ formation in MuSK $\triangle C R D$ mice suggest the use of Gsk3 inhibitors as potential therapeutic reagents to counteract neuromuscular disorders linked to Wnt/MuSK pathway deficiency.

\section{Materials and Methods}

All experiments on mice were performed in accordance with European Community guidelines legislation and reviewed by the local ethical committee of the Paris Descartes University (CEEA34.LS.030.12). The investigators had valid licenses (A-75-1970) to perform experiments on live vertebrates delivered by Direction des Services Vétérinaires (Préfecture de Police, Paris). The mutant line was established on the C57BL/6 genetic background, and experimental procedures were performed on mutant males and wild-type (WT) littermates.

\section{Generation of MuSK $\Delta$ CRD mutant mice and genotyping}

The MuSK ${ }^{\triangle C R D / \triangle C R D}$ mutant mouse line, lacking MuSK 315-478 amino acids corresponding to the CRD, was established at the Mouse Clinical Institute using proprietary vector containing floxed neomycin resistance cassette and protamine-Cre cassette (Illkirch, France; http://www-mci. $\mathrm{u}$-strasbg.fr). The use of protamine cassette in the construction vector offers an efficient solution for auto-excision of the floxed region when chimaera mice were bred with Cre-expressing mice. The targeting vector was constructed by successive cloning of PCR products and contained a $5.5 \mathrm{~kb}$ fragment (corresponding to the $5^{\prime}$ homology arm), a $4 \mathrm{~kb}$ floxed fragment including protamine Cre and neomycin selection cassettes, and a $5 \mathrm{~kb}$ fragment (corresponding to the $3^{\prime}$ homology arms). Two LoxP sequences delimiting the floxed fragment were located upstream of exon 9 and downstream of exon 11. The linearized construct was electroporated in BALB/CN mouse embryonic stem (ES) cells. Targeted ES clones were screened by $5^{\prime}$ external and Protamine Cre (inside the targeting vector) long-range PCR and by external and internal Southern blot. Two positive ES clones were injected into C57BL/6N blastocysts, and derived male chimaeras gave germline transmission. The resulting lines were crossed with a Cre deleter mouse generated on a pure inbred C57BL/6N background, in which the CRE gene is driven by the chicken $\beta$-actin promoter, and showing high and stable recombination efficiency to induce deletion of the floxed region (Birling et al., 2012). The following primers were used for PCR validation of the injected clones: 5' external
PCR, Efl (forward) 5'ATTTGAAATGCTGATTTGGATCCAT3' and Nr (reverse) 5'GCGGCCGGAGAACCTGCGTGCAATC3'; protamine Cre PCR, Cref (forward) 5'GGTAACTAAACTGGTCGAGCGATGG3' and Er (reverse) 5'ATGGATCCAAATCA GCATTTCAAAT3'. A 3' external probe (forward, 5'GGCGTAATGGAAGAAATAATCCT CT3'; reverse, 5'GACTGCAGAATCAGAAGGC3') with Drd1 restriction enzyme and a neo probe with ApaL1, EcoR1, Drd1, and Xcm 1 restriction enzymes were used for external and internal Southern blot analyses. Genotyping was performed in a single PCR reaction with a three primer set to detect both WT and mutated alleles. The following primers were used for genotyping: for the WT allele, Ef (forward) 5'CTC TTC TCC CTT CTG CCC ACC GAT3' and Wr (reverse) 5'CCC TGG GAA TAT GGT TTC TCA TTG CT3'; for the MuSK $\triangle C R D$ allele, Ef (forward) and Lxr (reverse) 5'AGT TAT ACT AGA GCG GCC GTT CAC CG3'. The WT amplified sequence was 234 bp long, whereas the knock-out amplification was 200 bp long.

\section{Antibodies}

The following antibodies were used: polyclonal and monoclonal Alexa Fluor 488-conjugated (Invitrogen, 1/1000), polyclonal Cy3 conjugated (Jackson ImmunoResearch Laboratories, 1/1000), monoclonal and polyclonal peroxidase-conjugated (GE Healthcare, 1/10,000), rabbit monoclonal anti-synaptophysin (Syn) (Invitrogen, 1/5), polyclonal antineurofilament (NF) $68 \mathrm{kDa}$ (Millipore Bioscience Research Reagents, $1 / 1000)$, polyclonal anti-NF $165 \mathrm{kDa}$ (DSHB, 1/750), polyclonal anti-HA (1/2500; Abcam), and monoclonal anti- $\beta$-catenin (Invitrogen, $1 / 500$ ). Polyclonal anti-MuSK (Abcam, 1/200), monoclonal anti-transferrin receptor (Invitrogen, 1/500), and anti- $\alpha$-tubulin (Sigma-Aldrich, 1/6000) were used for Western blot. Polyclonal anti-MuSK (1/500) used for immunohistochemistry and immunoprecipitation is a gift from M. Ruegg (Basel, Switzerland). DAPI $(1 / 20,000)$ and $\alpha$-BTX Alexa Fluor 488 conjugate $(1 / 1000)$ were purchased from Euromedex and Invitrogen, respectively.

\section{Plasmids}

The rat MuSK-HA and MuSK $\Delta$ CRD-HA cDNA plasmids have been previously described (Cartaud et al., 2004; Strochlic et al., 2012).

\section{Biotinylation of cell surface MuSK and MuSKDCRD,} immunoprecipitation, and Western blot analyses

HEK293T cells (ATCC) were cultured in DMEM supplemented with $10 \%$ FBS, 2 mm glutamine, and 2\%penicillin/streptomycin (500 U) at $37^{\circ} \mathrm{C}$ in $5 \% \mathrm{CO}_{2}$. Cells were grown to $70 \%$ confluence and transfected $(2$ $\mu \mathrm{g}$ of plasmids) using Fugen (Promega) transfection technique. Fortyeight hours after transfection, biotinylation of cell surface proteins and Western blot were performed as described previously (Sigoillot et al., 2010). The membrane transferrin receptor was used as a loading control to normalize the results. Relative signal intensity of total and cell surface MuSK-HA or MuSK $\Delta$ CRD-HA proteins was measured using ImageJ software. The levels of MuSK-HA and MuSK $\Delta$ CRD-HA in total extracts were normalized to $\alpha$-tubulin signals.

MuSK immunoprecipitation was performed from mutant or heterozygote E18.5 hindlimb muscles ( $N=4$ for each genotype) as described previously (Punga et al., 2011).

\section{Muscle primary cultures}

Muscle cells were isolated from P7-P10 hindlimb tibialis anterior (TA) and gastrocnemius muscles from MuSK $\Delta C R D$ or WT mice. Briefly, muscle tissues were excised, separated from connective tissue, minced in dissecting medium (DMEM-F12 medium containing $2 \mathrm{~mm}$ glutamine, $2 \%$ penicillin/streptomycin (500 U), $2 \%$ Fungizone), and dissociated in dissecting medium containing $0.2 \%$ Type I collagenase (Invitrogen ) for $90 \mathrm{~min}$ at $37^{\circ} \mathrm{C}$. Cells were centrifugated, filtered, and resuspended in proliferating medium (dissecting medium supplemented with $20 \%$ horse serum and $2 \%$ Ultroser G, Pall). After overnight preplating, cells were expanded on Matrigel-coated dishes (Corning) for 3-5 d and differentiated in differentiating medium (dissecting medium supplemented with $2 \%$ horse serum) for $5 \mathrm{~d}$. When indicated, myotubes were treated with recombinant agrin $(0.4 \mu \mathrm{g} / \mathrm{ml}$, R\&D Systems), Wnt11 (10 ng/ml, R\&D Systems), or LiCl (2.5 mm, Sigma) for $16 \mathrm{~h}$. 


\section{CT scan analysis and measurement of the kyphotic index (KI)}

Micro-CT scan analysis was performed in collaboration with the imaging platform PIPA installed in the imaging laboratory of EA 2496 (Montrouge). P90 WT and MuSK $\Delta C R D$ mice were sedated using 1.5\% isoflurane in air (TEC 3, Anestéo France). Entire body in dorsal and ventral decubitus of each animal was scanned by Quantum FX PerkinElmer micro-CT device (Caliper Rikagu) in dynamic mode. A tube voltage of 90 $\mathrm{kV}$ and a tube current of $160 \mu \mathrm{A}$ were selected. Total scan time was $2 \times$ $17 \mathrm{~s}$ per total animal scan. The scan field of view was $2 \times 60 \mathrm{~mm}$ with a spatial resolution of 118 micros (voxel size). Each scan was achieved by optimizing the resolution/dose ratio. Therefore, for the resolution selected, each animal was exposed to a low dose of $26 \mathrm{mGy}$. Image reconstructions and measurements were performed by the Osirix software version 5.6. The KI was determined from the direct multiplanar reconstructions as previously described (Laws and Hoey, 2004). Briefly, the distance (D1) from the seventh cervical vertebra (C7) to the sixth lumbar vertebra (L6) and then the perpendicular distance (D2) from D1 to the point of maximum vertebra curvature were measured. KI corresponds to the ratio D1/D2. KI is inversely proportional to kyphosis.

\section{Rail grip and grip strength measurements}

Animals were placed on a suspended metal rail, and their latency to release the rail was recorded. Each mouse ( $N=6$ for each genotype) was subjected to five trials with at least $10 \mathrm{~min}$ rest period between tests. The same group of mice was tested for the grip test assay at different time points. The grip strength was measured using a grip force tensiometer (Bioseb) according to the TREAT-NMD guidelines. Forelimb and hind limb traction strength was recorded following the manufacturer's instructions. Three measurements were performed per animal.

\section{Ex vivo isometric tension analyses}

$\mathrm{P} 120 \mathrm{WT}$ and MuSK $\triangle C R D$ mice were killed by dislocation of the cervical vertebrae followed by immediate exsanguination. Left hemidiaphragm muscles with their respective associated phrenic nerves were mounted in a silicone-lined bath filled with Krebs-Ringer solution of the following composition: $154 \mathrm{~mm} \mathrm{NaCl}, 5 \mathrm{~mm} \mathrm{KCl}, 2 \mathrm{~mm} \mathrm{CaCl} 2,1 \mathrm{~mm} \mathrm{MgCl}_{2}, 11 \mathrm{~mm}$ glucose, and $5 \mathrm{~mm}$ HEPES (buffered at $\mathrm{pH} 7.4$ with $\mathrm{NaOH}$ ), continuously perfused with $\mathrm{O}_{2}$ at $23.1 \pm 0.4^{\circ} \mathrm{C}$. One of the hemidiaphragm tendons (at the rib side) was securely anchored onto the silicone-coated bath via stainless steel pins while the other tendon was tied with silk thread, via an adjustable stainless steel hook, to an FT03 isometric force transducer (Grass Instruments). Muscle twitches and tetanic contractions were evoked by stimulating the motor nerve via a suction microelectrode adapted to the diameter of the nerve, with supramaximal current pulses of $0.15 \mathrm{~ms}$ duration, at frequencies indicated in the text. For each preparation investigated, the resting tension was adjusted at the beginning of the experiment (to obtain maximal contractile responses) and was monitored during the whole duration of the experiment. Signals from the isometric transducer were amplified, collected, and digitized with the aid of a computer equipped with an analog to digital interface board (Digidata 1200, Molecular Devices) using Axoscope 9 software (Molecular Devices).

\section{Electron microscopy}

P120 WT and MuSK $\triangle C R D$ mice were sedated using 1.5\% isoflurane in air (Minerve Equipement vétérinaire). TA muscles were then dissected and immediately fixed in 2\% glutaraldehyde and 2\% PFA in PBS for $1 \mathrm{~h}$ at room temperature and overnight at $4^{\circ} \mathrm{C}$. TA muscles were rinsed in water, and AChE staining following Koelle's protocol was performed. The endplate-containing tissue blocks were cut in small pieces. Subsequently, tissue samples were washed three times in $0.1 \mathrm{M}$ sodium phosphate buffer, incubated $30 \mathrm{~min}$ at $4^{\circ} \mathrm{C}$ in $1 \%$ osmic acid in sodium phosphate buffer, and dehydrated in graded ethanol solutions $(2 \times 10$ min in $70 \%, 2 \times 10 \mathrm{~min}$ in $90 \%$ and $2 \times 10 \mathrm{~min}$ in $100 \%$ ethanol). Samples were then incubated twice in propylene for $1 \mathrm{~min}, 10 \mathrm{~min}$ in $50 \%-50 \%$ Epon- $50 \%$ propylene oxide, then embedded in Epon and incubated for polymerization $24 \mathrm{~h}$ at $60^{\circ} \mathrm{C}$. The $90 \mathrm{~nm}$ sections were cut on a Reichert Ultracut S and laid on a grid for staining 10 min with $2 \%$ uranyl acetate and $4 \mathrm{~min}$ with lead citrate at room temperature. The observations were performed with a JEOL 1011 transmission electron microscope and the images recorded at $80 \mathrm{kV}$ with a GATAN Erlangshen 1000 camera.

\section{Biochemical analyses of acetylcholinesterase activity}

Frozen diaphragm and soleus muscles from P90 WT and MuSK $\Delta C R D$ mice were homogenized in a protein extraction buffer $(50 \mathrm{~mm}$ phosphate buffer, pH 7.6, $1 \%$ chaps, $0.8 \mathrm{M} \mathrm{NaCl}$, and $10 \mathrm{~mm}$ EDTA) completed with a protease inhibitors mixture (Roche). Total AChE activity in the extracts was assayed using $0.7 \mathrm{~mm}$ acetylthiocholine and $0.5 \mathrm{~mm} \mathrm{5,5-}$ dithiobis(2-nitrobenzoic acid) (DTNB) in the presence of $50 \mu \mathrm{M}$ tetra(monoisopropyl)-pyrophosphortetramide (Sigma). The extracts were first incubated in the absence of acetylthiocholine for at least $20 \mathrm{~min}$ to block butyrylcholinesterase and saturate the free sulfhydryl groups that interact with DTNB. The change in optical density was measured at $414 \mathrm{~nm}$.

\section{Immunohistochemistry}

Whole-mount diaphragm staining. Diaphragm muscles were dissected and fixed (4\% PFA in PBS) for $1 \mathrm{~h}$ at room temperature and further fixed ( $1 \%$ formaldehyde in PBS) overnight at $4^{\circ} \mathrm{C}$. Muscles were washed three times for $15 \mathrm{~min}$ in PBS, incubated for $15 \mathrm{~min}$ with $100 \mathrm{~mm}$ glycine in PBS, and rinsed in PBS. Muscles were permeabilized (0.5\% Triton X-100 in PBS) for $1 \mathrm{~h}$ and blocked for $4 \mathrm{~h}$ in blocking buffer (3\% BSA, 5\% goat serum and $0.5 \%$ Triton $\mathrm{X}-100$ in PBS). Muscles were incubated overnight at $4^{\circ} \mathrm{C}$ with rabbit polyclonal antibodies against NF and Syn in blocking solution. After three $1 \mathrm{~h}$ washes in PBS, muscles were incubated overnight at $4^{\circ} \mathrm{C}$ with Alexa Fluor 488 goat anti-rabbit IgG and Alexa Fluor 488-conjugated $\alpha$-BTX in blocking solution. After three $1 \mathrm{~h}$ washes in PBS, muscles were flat-mounted in Vectashield (Vector Laboratories) mounting medium.

Tissue section staining. Dissected TA muscles from $\mathrm{P} 90$ adult mice were fixed ( $1 \% \mathrm{PFA}$ in PBS) for $1 \mathrm{~h}$ at $4^{\circ} \mathrm{C}$, rinsed twice at $4^{\circ} \mathrm{C}$ in PBS, cryoprotected (30\% sucrose in PBS) overnight at $4^{\circ} \mathrm{C}$, embedded in TissueTek (Sakura), and quickly frozen in isopentane cooled in liquid nitrogen. Cryostat cross sections $(12 \mu \mathrm{m}$ ) were permeabilized with $0.5 \%$ Triton X-100 in PBS for 10 min and labeled with various antibodies as for whole-mount immunostaining. Incubation times were the following: $1 \mathrm{~h}$ for blocking, overnight at $4^{\circ} \mathrm{C}$ for incubation with primary antibodies and $1 \mathrm{~h}$ for incubation with secondary antibodies.

Isolated muscle fiber staining. Dissected and isolated TA muscle fibers from P20, P40, and P60 adult mice were fixed (4\% PFA in PBS) for 30 min at $4^{\circ} \mathrm{C}$ and rinsed with $\mathrm{PBS}$ at room temperature. Isolated muscle fibers were labeled with antibodies as for whole-mount immunostaining. Incubation times were the following: $4 \mathrm{~h}$ for blocking, overnight at $4^{\circ} \mathrm{C}$ for incubation sucessively with primary or secondary antibodies.

\section{Images acquisition and processing}

All images were collected on a microscope (model BX61; Olympus) equipped with a Fast 1394 Digital CCD FireWire camera (model Retiga 2000r; Qimaging) and a 20× objective or on a confocal laser scanning microscope (Zeiss, LSM-710) equipped with a $20 \times$ objective and $63 \times$ oil objective. Collected $Z$-stack confocal images (5-20 stacks with 1-1.5 $\mu \mathrm{m}$ $(20 \times) z$-steps) and image capture were made using LSM Image Browser. The same laser power and parameter setting were applied to ensure reliable comparison between WT and MuSK $\triangle C R D$ muscles. Multiple tile scanned images were taken to cover the size of the whole diaphragm. Confocal images presented are single-projected image derived from overlaying each set of stacks. For quantification of the AChR cluster number, volume, and intensity, image stacks were quantified using the ImageJ (version $1.46 \mathrm{~m}$ ) plugin "3D object counter" (Bolte and Cordelières, 2006). The threshold intensity was set by visual inspection of AChR clusters, being the same between WT and MuSKDCRD images. The endplate band width was defined by the distance between the two farthest AChR clusters from the main nerve trunk. Approximately 100 measurements regularly spaced and covering the entire diaphragm were taken. At least 4 diaphragms or 50 isolated muscle fibers of each genotype were analyzed and quantified. To evaluate $\beta$-catenin translocation to subsynaptic nuclei in isolated muscle fibers, image stacks corresponding to nuclei were used for quantification using the ImageJ intensity plot 
profile measuring the intensity of $\beta$-catenin and DAPI fluorescence within the segmented line.

Neonatal and pregnant mice intraperitoneal injections

E12 pregnant mice or $\mathrm{P} 10$ adult mice were intraperitoneally injected with $\mathrm{LiCl}$ (Sigma, $600 \mathrm{~mm} 10 \mu \mathrm{l} / \mathrm{g}$ body weight) or placebo $\mathrm{NaCl}$ solution $(0,0009 \% 10 \mu \mathrm{l} / \mathrm{g}$ body weight $)$. Daily injections were made from E12 to E18 or from P10 to P60. After embryos genotyping, E18.5 or P60 diaphragms of LiCl-treated MuSK $\triangle C R D$ mice were analyzed and compared with WT or NaCl-treated MuSK $\triangle C R D$.

\section{Statistical analysis}

Data are expressed as mean \pm SEM. Statistical analyses and graphs were performed with Prism 6.0 (Graphpad) software. All data were analyzed using the Mann-Whitney $U$ test or two-way ANOVA, wherever appropriate ( $p<0.05$ considered significant). Each experiment was conducted a minimum of three times.

\section{Results}

\section{Generation of MuSKDCRD transgenic mice}

We generated a mouse line deleted from the MuSK CRD by homologous recombination (Fig. 1A). To construct the MuSKCRD floxed allele, we created a targeting vector consisting of the MuSK gene flanked by two loxP sites upstream of exon 9 and downstream of exon 11. The construct was electroporated in $\mathrm{BALB} / \mathrm{CN}$ mouse ES cells, and the injected clones were controlled by PCR (5' external and Protamine Cre PCR) followed by internal (neo probe with ApaL1 or Drd1) and external ( $3^{\prime}$ external probe with Drd1 restriction enzyme) Southern blot analyses to confirm $5^{\prime}$ and $3^{\prime}$ correct integration and absence of second random integration (Fig. $1 B$ ). Two positive and independent ES cell clones were injected into $\mathrm{C} 57 \mathrm{BL} / 6 \mathrm{~N}$ blastocysts to generate two independent $M u S K^{f l o x(C R D)}$ mice. These mice were then crossed with Cre deleter mice to generate offspring with MuSK CRD deletion (see Material and Methods; Fig. 1C for genotyping). A single band corresponding to MuSK deleted from its CRD $(85 \mathrm{kDa})$ was detected in E18.5 MuSK $\triangle C R D$ hindlimb muscles after MuSK immunoprecipitation confirming that the CRD deletion occurred in the mutant mice (Fig. 1D). Both heterozygous $M u S K^{+/ \Delta C R D}$ and homozygous $M u S K^{\triangle C R D / \triangle C R D}$ (MuSKDCRD) mutant mice were indistinguishable from WT mice. Although $5 \%$ of the MuSK $\triangle C R D$ mice died a few days after birth, being smaller in weight and size, exhibiting respiratory failure as well as limb motor deficiency, most of them were viable, able to suck milk, and developed up to adulthood with normal fertility. In addition, no difference in the weight curve between MuSK $\triangle C R D$ and WT mice could be detected (data not shown).

Given that deletion of MuSK CRD could lead to a deficit of the mutated MuSK expression at the cell membrane, we quantified the level of membrane WT or mutated MuSK using in vitro biotinylation experiments and tissue immunohistochemistry. Labeling of surface proteins by biotinylation in HEK293T cells expressing MuSK-HA or MuSK $\Delta$ CRD-HA showed similar levels of MuSK and MuSK $\Delta$ CRD at the plasma membrane (Fig. 1E). Moreover, both WT and mutated MuSK colocalized with membrane AChRs clusters labeled with $\alpha$-BTX in P60 WT and MuSK $\triangle C R D T A$, indicating that deletion of MuSK CRD does not disrupt the mutated MuSK membrane localization at the NMJ (Fig. $1 F$ ). Quantification of the level of WT and mutated MuSK signal intensity at the synapse showed an increase of $70 \%$ in $M u S K \triangle C R D$ compared with WT mice (Fig. $1 G$ ). In addition, because MuSK localization at the synapse is required for anchoring AChE in the postsynaptic membrane (Cartaud et al., 2004), we asked whether AChE localization and activity were disturbed in $M u S K \triangle C R D$ muscles. Histochemical staining revealed that
AChE was accumulated in the postsynaptic membrane of both WT and MuSK $\Delta C R D P 90$ TA muscles (Fig. $1 H$ ). No difference in AChE activity could be detected between WT and MuSK $\triangle C R D$ muscles (Fig. 1I).

To test whether deletion of MuSK CRD could disturb agrinLrp4-MuSK signaling, we quantified agrin-induced AChR clusters in WT and MuSK $\triangle C R D$ primary muscle cultures. No difference in the number of AChR clusters following agrin treatment was detected in MuSK $\triangle C R D$ compared with WT primary myotubes, indicating that MuSK CRD deletion does not perturb agrin-induced AChR clustering. These data suggest that agrin is able to interact with Lrp4 in the Lrp4/MuSK $\Delta$ CRD complex and that MuSK $\Delta \mathrm{CRD} / \mathrm{Lrp} 4$ interaction can transduce downstream signaling (Fig. $1 \mathrm{~J}, \mathrm{~K}$ ).

\section{Deletion of MuSK CRD affects NMJ formation}

Given that Wnts are known to play a role during the early steps of NMJ formation through binding to MuSK CRD (Jing et al., 2009; Strochlic et al., 2012), we tested whether deletion of MuSK CRD could lead to NMJ defects during development. First, we compared NMJ phenotype in E14 WT and MuSK $\triangle C R D$ embryos (Fig. 2). Whole-mount diaphragms were stained with $\alpha$-BTX to detect AChR clusters and with a mixture of antibodies against neurofilament (NF) and synaptophysin (Syn) to label axonal branches and nerve terminals, respectively. Both dorsal and ventral portions of each hemidiaphragm were innervated, indicating that axonal extension is fully developed in MuSK $\triangle C R D$ embryos (Fig. 2A). Nonetheless, the neurites were increased in length by $82 \%$ (Fig. $2 B, C$ ). In addition to this presynaptic defect, AChR clustering was also affected in MuSK $\Delta$ CRD embryos. In WT embryos, AChR clusters were concentrated in the central zone of the muscle as expected during prepatterning. In contrast, in mutant embryos, AChR clusters were almost undetectable and were distributed in a twofold wider muscle area (Fig. $2 B, D$, arrowheads). Quantitative analysis revealed a mean 64\% decrease in the number of MuSK $\triangle C R D$ AChR clusters (Fig. 2E). Moreover, $M u S K \triangle C R D$ AChR clusters volume and intensity were reduced by $70 \%$ and $65 \%$, respectively, and non-innervated AChR clusters were increased by $30 \%$ in MuSK $\triangle C R D$ compared to WT embryos (Fig. $2 F-H$ ). These results suggest that deletion of MuSK CRD leads to an early developmental defect of the NMJ with a severely reduced AChR prepatterning.

We further analyzed NMJ morphology later during development in E18.5 MuSK $\Delta C R D$ diaphragms (Fig. 3). Whereas AChR clusters were tightly restricted to a thin endplate band in WT hemidiaphragms, the endplate band width was twofold larger in MuSK $\triangle C R D$ (Fig. $3 A, B$ ). Furthermore, AChR clusters were reduced in number by $40 \%$ (Fig. $3 C$ ), volume by $25 \%$ (Fig. $3 D$ ), and intensity by $14 \%$ (Fig. $3 E$ ). All AChR clusters were innervated in WT and MuSK $\Delta C R D$; however, MuSK $\Delta C R D$ embryos showed aberrant extension of motor axons, bypassing AChR clusters and growing excessively toward the periphery of the muscle (Fig. $3 F$ ). Although the number of primary and secondary branches was not significantly affected, the length of primary as well as secondary branches was increased by $75 \%$ and $46 \%$, respectively, in $M u S K \Delta C R D$ mutants compared with WT embryos (Fig. 3G-J).

Together, these results indicate that MuSK CRD deletion drastically perturbs NMJ formation as exemplified by reduced prepatterned and neural AChR clusters as well as exuberant neurite outgrowth. 

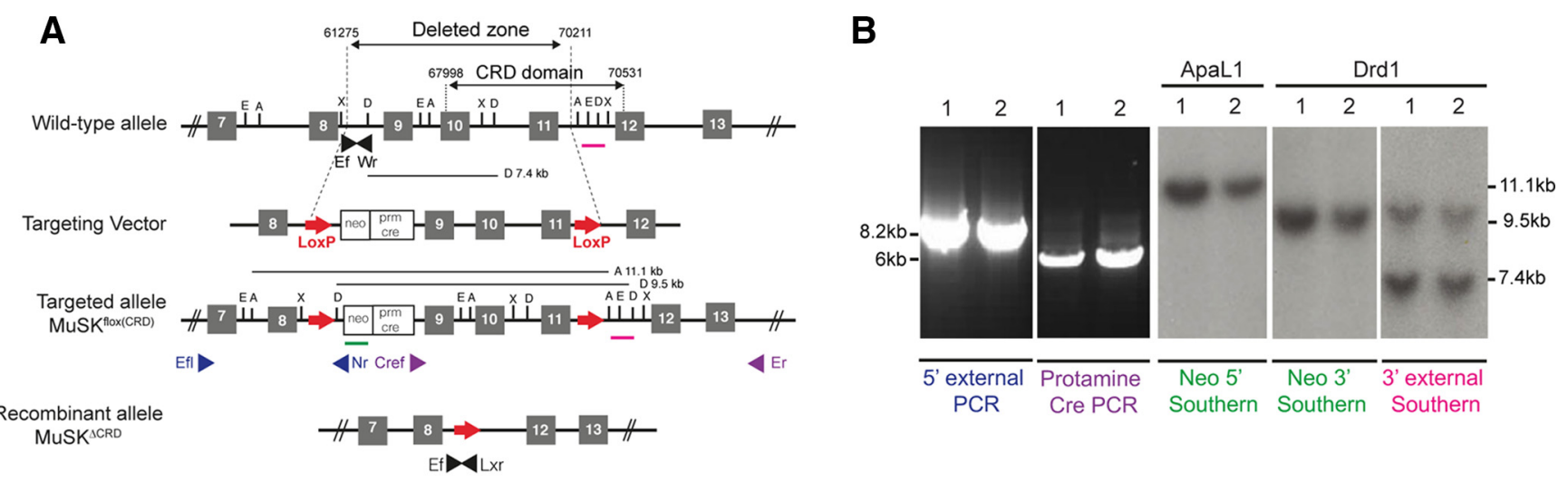

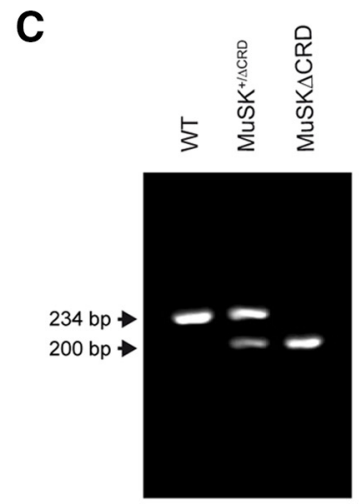

F
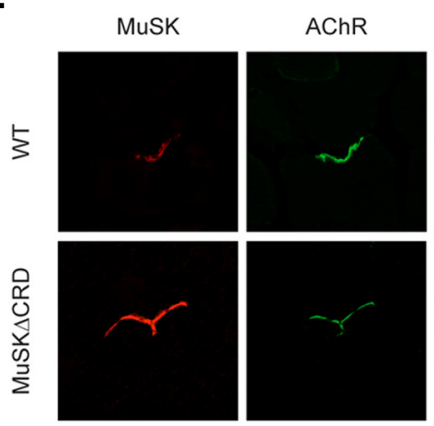

I

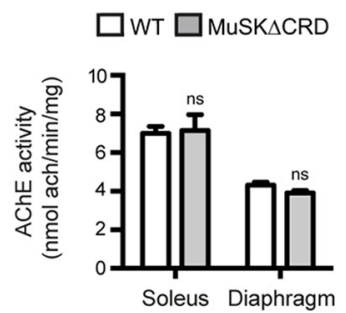

D

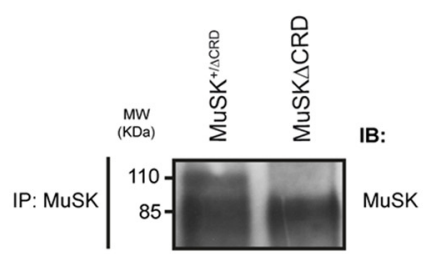

E

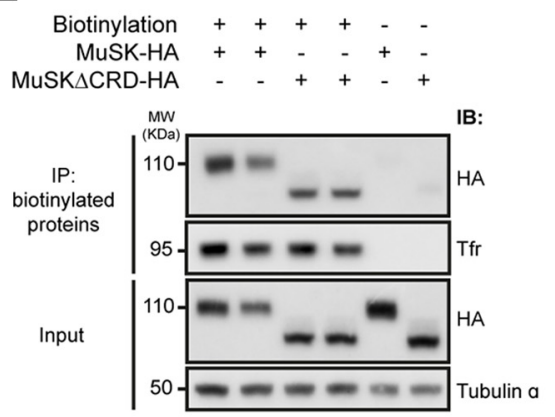

H

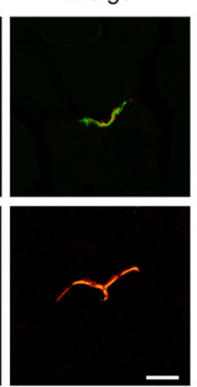

J
G

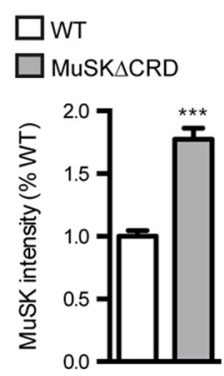

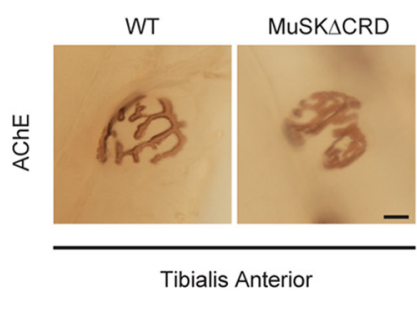

K

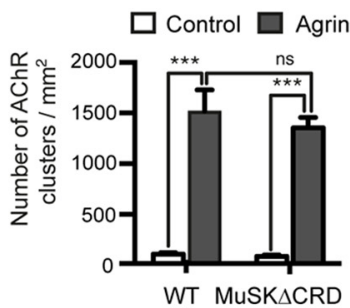

Figure 1. Generation of MuSK $\triangle C R D$ transgenic mice. $A$, Schematic representation of the KO strategy. First panel, Genomic structure of the MuSK gene encompassing MuSK CRD. Second panel,

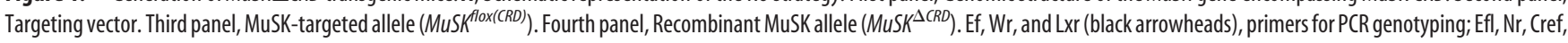
and Er, primers for PCR validation of the ES clones (blue arrowheads for $5^{\prime}$ external and violet arrowheads for protamine (re PCR). Pink line indicates 3' external Southern blot probe. Green line indicates neo internal Southern blot probe. A, ApaL1; D, Drd1; X, Xcm1. B, Examples of 5' external and protamine Cre PCR validation and internal and external Southern blot hybridization analyses of two injected ES mutant clones (lanes 1 and 2). Amplified bands of the expected size for PCR (8.2 and $6 \mathrm{~kb}$ ) and for $5^{\prime}$ internal (ApaL1, targeted band, $11.1 \mathrm{~kb}$ ), $3^{\prime}$ internal (Drd1, targeted band, $9.5 \mathrm{~kb}$ ), and 3' external (Drd1; WT band, $7.4 \mathrm{~kb}$; targeted band, $9.5 \mathrm{~kb}$ ) Southern blots were obtained. C, Genotyping by PCR using a three primer set. The 234 and 200 bp bands represent WT and MuSK $\Delta C R D$ alleles, respectively. D. Western blot of MuSK or MuSK $\Delta C R D$ using MuSK antibodies in E18.5 MuSK ${ }^{+/ \Delta C R D}$ or MuSK $\Delta C R D$ hindlimb muscle after MuSK immunoprecipitation. E, Western blot of cell surface and total MuSK-HA and MuSK $\Delta$ CRD-HA in HEK293T cells transfected with MuSK-HA or MuSK $\Delta$ CRD-HA. Transferrin receptor (Tfr) and $\alpha$-tubulin were used as a loading control for biotinylated proteins and input, respectively. Transfection of MuSK-WT-HA or MuSK $\triangle$ CRD-HA was performed in duplicate. $F$, Confocal images of P60 WT or MuSK $\triangle$ CRD TA muscle cross sections stained with MuSK antibody (red) together with $\alpha$-BTX (AChR, green). G, Quantification of WT and mutated MuSK signal intensities at the synapse. $\boldsymbol{H}$, Koelle's histochemical staining of AChE performed on isolated TA muscle fibers from P90 WT and MuSK $\triangle C R D$. I, Quantification of AChE activity extracted from P90 WT and MuSK $\triangle C R D$ soleus and diaphragm. J, Examples of myotubes isolated from WT or MuSK $\triangle C R D$ primary cultures, treated or not with recombinant agrin and stained with $\alpha$-BTX. $K$, Quantitative analysis of the number of AChR clusters in WT and MuSK $\Delta C R D$ myotubes. Data are mean \pm SEM. ${ }^{* * *} p<0.001$. ns, Not significant. $N=3$ independent experiments. Scale bar: $\boldsymbol{F}$ (merged image), $\boldsymbol{J}, 20 \mu \mathrm{m} ; \boldsymbol{H}, 50 \mu \mathrm{m}$. 
A
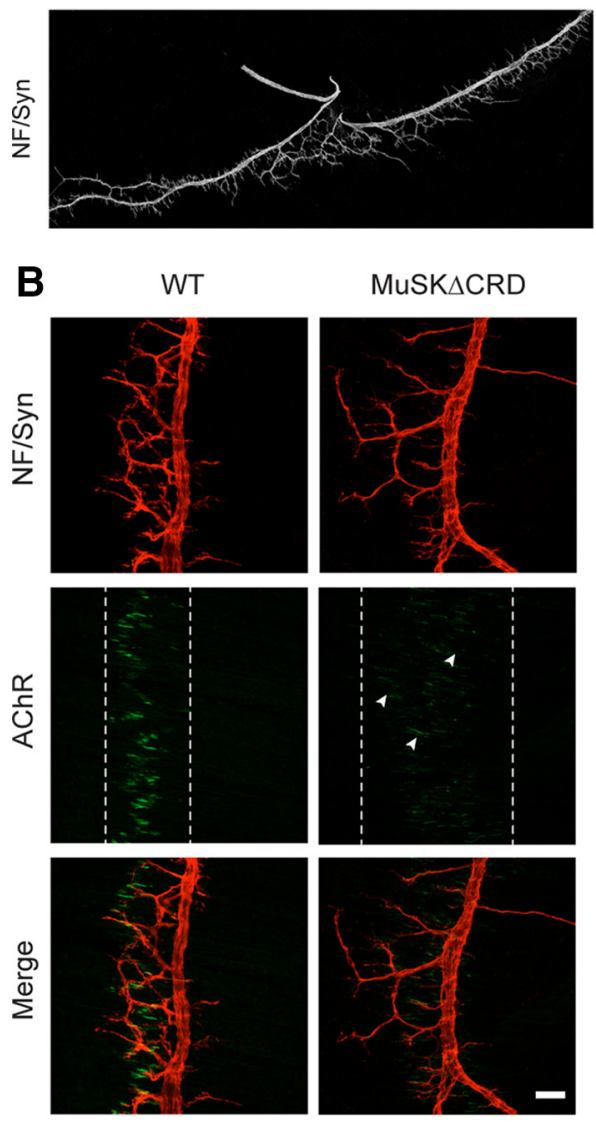

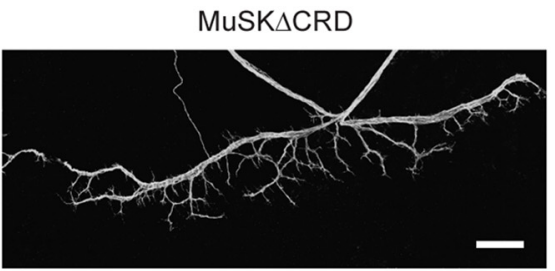

C
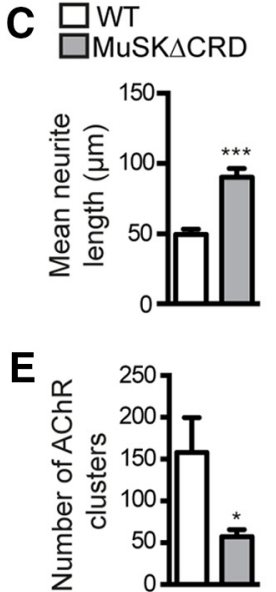

G

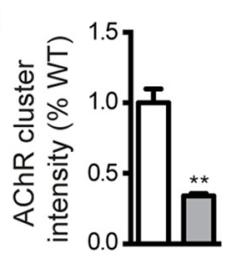

D 吕WT

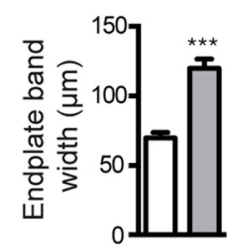

$\mathbf{F}$

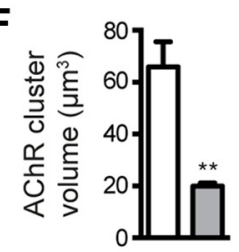

H

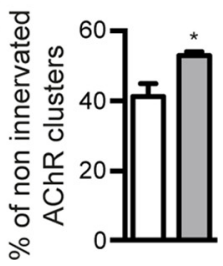

Figure 2. Impaired muscle prepatterning in MuSK $\triangle C R D$ embryos. $A, B$, Confocal images of whole-mount left hemidiaphragms from E14 WT and MuSK $\Delta C R D$ embryos stained with NF (red) and Syn (red) antibodies $(\boldsymbol{A}, \boldsymbol{B})$ together with $\alpha$-BTX (AChRs, green, $B$ ). Arrowheads indicate AChR clusters. White dashed lines indicate the synaptic endplate band and include most AChR clusters. $\boldsymbol{C}-\boldsymbol{H}$, Quantitative analysis of the mean neurite length $(\boldsymbol{C})$, the endplate band width $(\boldsymbol{D})$, the AChR cluster number $(\boldsymbol{E})$, volume $(\boldsymbol{F})$, intensity $(\boldsymbol{G})$, and non-innervated $A C h R$ clusters $(\boldsymbol{H})$. Number of AChR clusters analyzed: 790 in WT and 461 in MuSKACRD. Data are mean \pm SEM. ${ }^{*} p<0.05$ (Mann-Whitney U test). ${ }^{* *} p<0.01$ (Mann-Whitney U test). ${ }^{* *} p<0.001$ (Mann-Whitney U test). $N=4$ embryos per genotype. Scale bar: $\boldsymbol{A}, 300 \mu \mathrm{m} ; \boldsymbol{B}$ (merged image), $50 \mu \mathrm{m}$.

\section{$M u S K \triangle C R D$ adult mice exhibit immature and fragmented NMJs}

As previously mentioned, $M u S K \Delta C R D$ mutant mice are viable at birth. Therefore, we wondered whether NMJ defects observed during development would be also detected in newborn and adult mutant mice. NMJ phenotype analyzed in P5 WT and $M u S K \triangle C R D$ whole-mount preparations of diaphragm revealed AChR cluster deficit and neurite outgrowth defects in $M u S K \triangle C R D$ similar to those observed in MuSK $\triangle C R D$ embryos (Fig. 4A-E). To assess whether the presynaptic and postsynaptic counterparts were still affected in $M u S K \Delta C R D$ adult mice, we analyzed NMJ morphology on isolated muscle fibers from WT and MuSK $\Delta C R D$ TA muscles at P20 and P60 (Fig. 5). At birth, the shape of the endplates is ovoid and as NMJs mature, the endplates begin to acquire their branched postnatal topology (Marques et al., 2000; Kummer et al., 2006). In P20 MuSKSCRD mice, most of the endplates analyzed were ovoid and compact compared with the perforated WT ones, suggesting that NMJs are immature (Fig. 5A, left). Both analyzed AChR endplates of WT and $M u S K \triangle C R D$ mice were innervated as confirmed by nerve terminal staining, which colocalized with AChR clusters (Fig. 5A, left). However, quantitative analysis revealed that the number of AChR clusters as well as nerve terminal area per NMJ were significantly reduced by $49 \%$ and $58 \%$, respectively, in MuSK $\triangle C R D$ mice compared with WT mice without affecting the final overlap area between presynaptic and postsynaptic elements (Fig. 5B-D).

At P60, WT NMJs formed a continuous branched postnatal topology and exhibited a typical "pretzel-like" structure (Fig. $5 A$, right). At this stage, $10 \%$ of the analyzed NMJs in MuSK $\triangle C R D$ were similar in shape to WT ones (data not shown). However, the structure of most MuSK $\triangle C R D$ synapses ( $90 \%$ of the analyzed NMJs) was severely altered with the following characteristics: the postsynaptic network was discontinuous and isolated AChR clusters were frequently observed, suggesting fragmentation of the NMJs. Indeed, the number of AChRs fragments per NMJ was increased by fourfold (Fig. $5 E)$. Moreover, the total occupied AChR clusters area per NMJ was significantly reduced by $40 \%$, suggesting a loss of AChRrich domains (Fig. 5F). Axonal branches appeared discontinuous and fragmented in correlation with the postsynaptic apparatus parceling. The nerve terminal area was reduced by $39 \%$ without affecting the overlap area between presynaptic and postsynaptic elements (Fig. 5G,H). Similar NMJ defects were observed in other muscle types including fast-twitch extensor digitorum longus and slow-twitch soleus (data not shown).

Together, our findings indicate that the CRD deletion of MuSK perturbs NMJs maturation that finally leads to a severe dismantlement of the postsynaptic and presynaptic apparatus in adult mice.

\section{MuSK $\Delta C R D$ mice display altered NMJ ultrastructures}

To analyze the morphological alterations of muscle and NMJs at the ultrastructural level in MuSK $\triangle C R D$ mice, we performed electron microscopic analysis on TA muscle of P120 WT and MuSK $\Delta C R D$ mice (Fig. $6 A-M$ ). Although presynaptic specialization of nerve terminals appeared normal in MuSK $\triangle C R D$ mice with abundant mitochondria in the axoplasm, quantification analyses revealed a $67 \%$ decrease in MuSK $\triangle C R D$ synaptic vesicle density compared to WT mice without affecting the mean synaptic vesicle diameter (Fig. $6 H, I)$. Moreover, on the postsynaptic side, $\sim 30 \%$ of MuSK $\triangle C R D$ NMJs exhibited highly disorganized or very few junctional folds (JFs) compared with WT mice (78\% decrease of JFs in MuSK $\Delta C R D$; Fig. $6 A-F, J)$. In some cases, compared to WT, the distance between JFs and muscle fibers was increased and abundant accumulation of mitochondria beneath the postsynaptic membrane was observed in MuSK $\Delta C R D$ mice (Fig. 6G). However, no morphological difference between WT and MuSKDCRD overall muscle structure could be detected (Fig. $6 \mathrm{~K}$ ). Indeed, the sarcomeric organization and the distance between $Z$-lines were similar between WT and $M u S K \Delta C R D$ mice (Fig. $6 L$ ). In addition, MuSK $\Delta C R D$ mice did not show any alteration in the myelin sheath diameter (Fig. 6M). 
Collectively, these results indicate that MuSK CRD deletion alters both presynaptic and postsynaptic ultrastructures, which may result in a motor activity deficit.

\section{MuSK $\Delta C R D$ mice progressively develop muscle weakness, fatigue, and motor defects}

During the first 2 weeks after birth, MuSK $\triangle C R D$ mice developed normally without any gross physical signs of muscle weakness compared with WT mice. After this period, changes in the trunk region became progressively evident by the abnormal spine curvature caused by the shrinkage of the pelvic and scapular region. The CT scan analysis performed on P90 WT and MuSK $\triangle C R D$ mice illustrated the kyphosis developed by MuSK $\triangle C R D$ mice (Fig. 7A). kyphotic index (KI; see Material and Methods) used to appreciate the kyphotic severity degree was significantly reduced in MuSK $\Delta$ CRD compared to $\mathrm{WT}$, confirming the presence of a severe spine deformation in mutant mice (Fig. 7B) (Laws and Hoey, 2004). To assess whether motor function was altered in $M u S K \triangle C R D$ mice, we performed a grip test assay on young and adult mice. Whereas the latency to fall from the rail increased with age in WT mice, MuSK $\triangle C R D$ mice exhibited poor motor performance from P20 to P90 as determined by a reduced latency to fall (latency decrease: P20, 62\%; P40, 33\%; P60, 64\%; P90, 72\%; Fig. 7C). To further investigate the origin of motor defect in MuSK $\triangle C R D$ mice, we measured the grip strength of the forelimbs (Fig. 7D) and the hindlimbs (Fig. 7E). In MuSK $\triangle C R D$ as in WT mice, the forelimb and the hindlimb grip strength increased with age. However, the grip strength of $M u S K \Delta C R D$ mice was reduced at all time points compared to WT mice, indicating a muscle weakness (forelimb grip strength decrease: P20, 26\%; P40, 23\%; P60, 20\%; P90, 30\%; hindlimb grip strength decrease: P20, 37\%; P40, 18\%; P60, 23\%; P90, 21\%; Fig. 7 D, E).

To confirm the occurrence of muscle weakness in MuSK $\triangle C R D$, we analyzed the ability of P120 WT and MuSK $\Delta C R D$ left hemidiaphragms to evoke twitches and tetanic contractions in response to phrenic nerve stimulation at different frequencies ex vivo. As shown in Figure $7 F$, WT as MuSK $\triangle C R D$ muscles developed and maintained tetanic contractions, in a wide range of nerve-stimulation frequencies. However, MuSK $\triangle C R D$ muscles developed less force than WT ones. Indeed, in MuSK $\triangle C R D$ mice, the strength
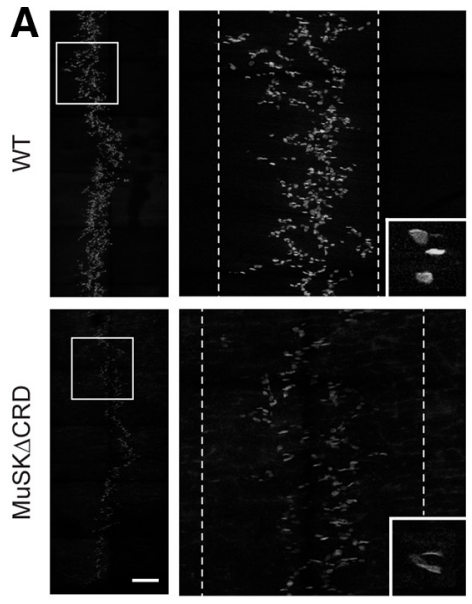

$\mathbf{F}$

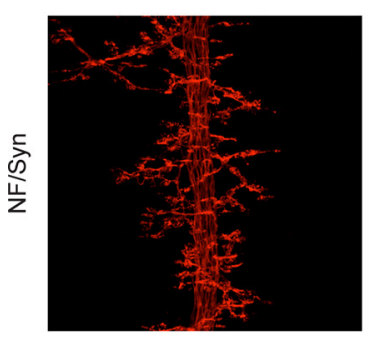

WT

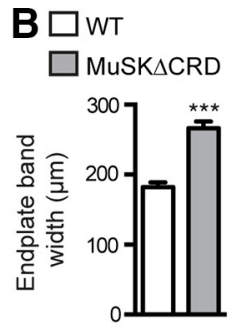

D

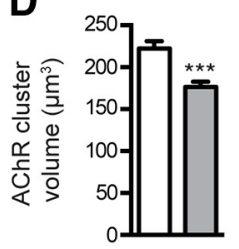

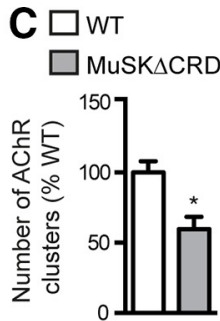

E

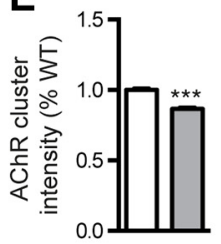

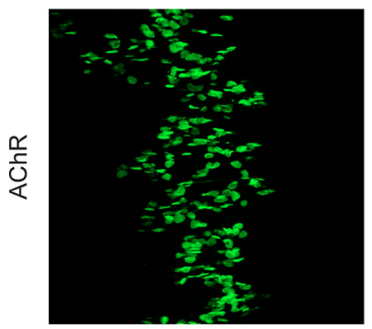
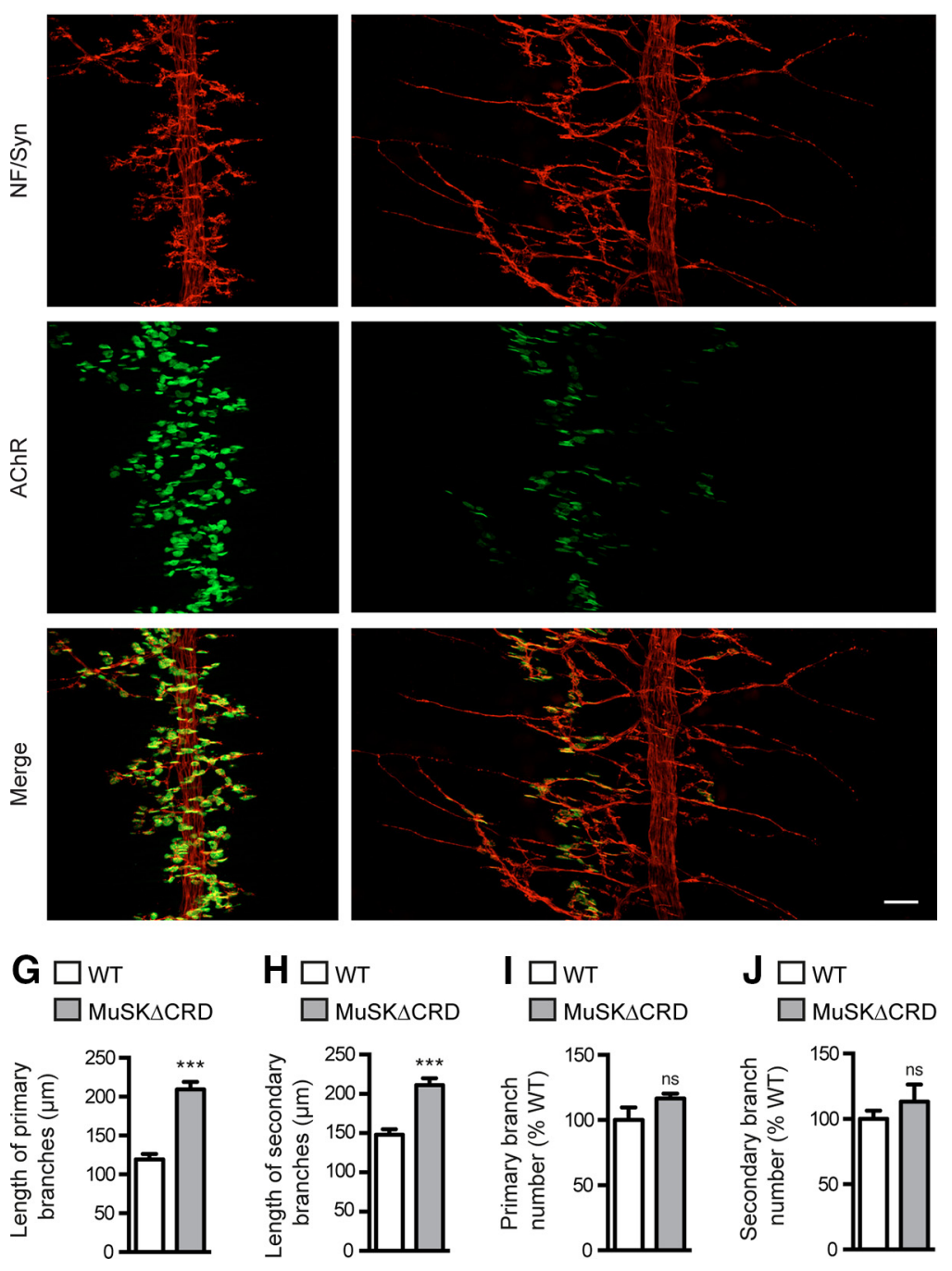

Figure 3. AberrantNMJ formation in E18.5 MuSK $\triangle$ CRD embryos. A, Confocal images of whole-mount left hemidiaphragms from E18.5 WT and MUSK $\triangle C R D$ embryos stained with $\alpha$-BTX to visualize AChR clusters. Right panels, Enlarged images of boxed regions in left panel. White dashed lines indicate the synaptic endplate band and include most AChR clusters. Right panels, Insets, Higher-magnification views of AChR clusters. $\boldsymbol{B}-\boldsymbol{E}$, Quantifications of the endplate band width $(\boldsymbol{B})$, the AChR cluster number $(\boldsymbol{C}$, volume $(\boldsymbol{D})$, and intensity $(\boldsymbol{E})$. Numbers of AChR clusters analyzed: 806 in WT and 604 in MUSK $\triangle$ CRD. $F$, Confocal images of whole-mount left hemidiaphragms from E18.5 WT and MuSK $\Delta$ CRD embryos stained as in Figure 2B. $\mathbf{G}-\boldsymbol{J}$, Quantitative analysis of the length $(\boldsymbol{G}, \boldsymbol{H})$ and the number $(\boldsymbol{I}, \boldsymbol{J})$ of primary and secondary nerve branches. Number of primary branches analyzed: 273 in WT and 309 in MuSK $\Delta C R D$; secondary branches: 266 in WT and 310 in MuSK $\Delta$ CRD. Data are mean \pm SEM. ${ }^{*} p<0.05$ (Mann-Whitney $U$ test). ${ }^{* *} p<0.01$ (Mann-Whitney $U$ test). ${ }^{* *} p<0.001$ (MannWhitney $U$ test). ns, Not significant. $N=6$ embryos per genotype. Scale bar, $\boldsymbol{A}, 300 \mu \mathrm{m} ; \boldsymbol{F}$ (merged image), $50 \mu \mathrm{m}$. 

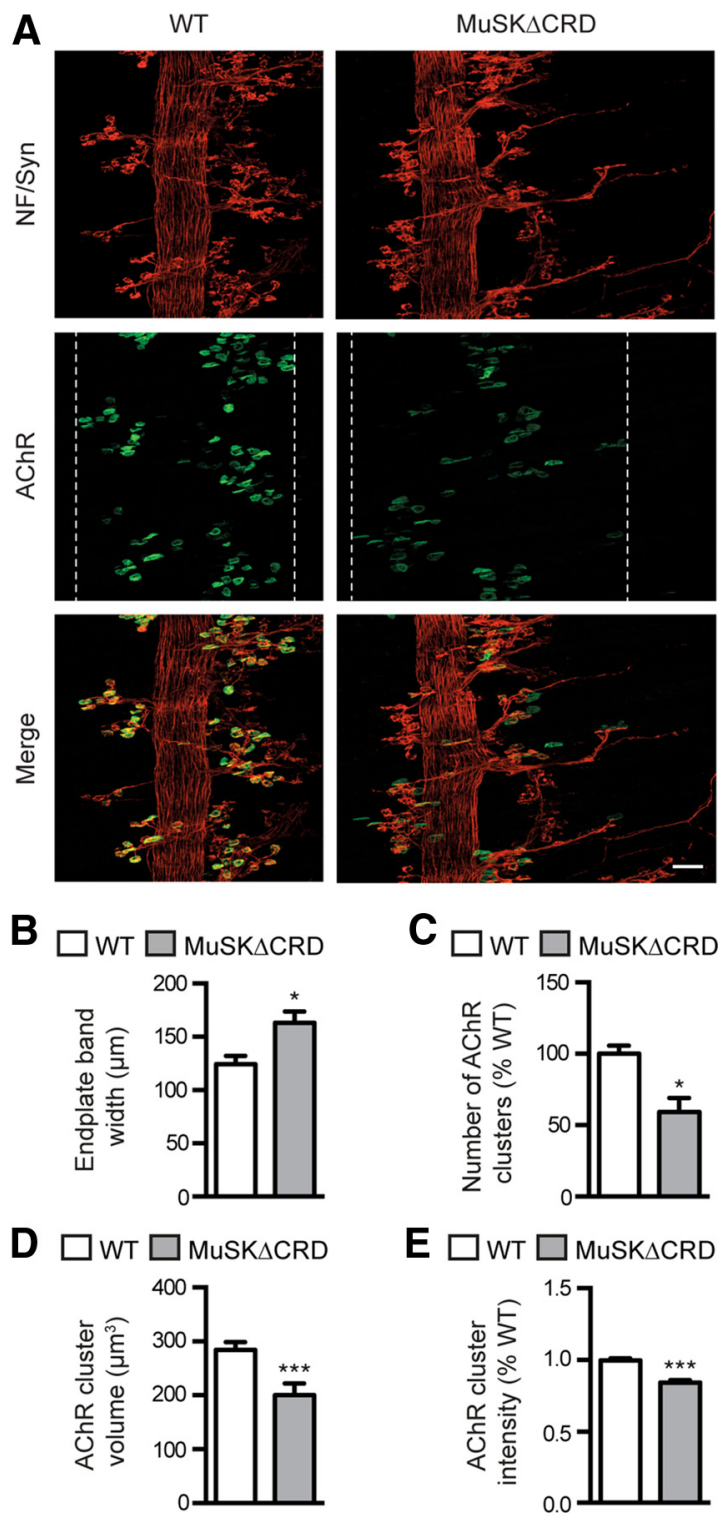

Figure 4. Diaphragm innervation defects in P5 MuSK $\triangle C R D$ mice. $A$, Confocal images of whole-mount P5 WT and MuSK $\Delta C R D$ left hemidiaphragms stained as Figure $2 B$. White dashed lines indicate the synaptic endplate band and include most AChR clusters. $\boldsymbol{B}-\boldsymbol{E}$, Quantitative analysis of the endplate band width $(\boldsymbol{B})$, the $A C h R$ cluster number $(\boldsymbol{C})$, volume $(\boldsymbol{D})$, and intensity $(\boldsymbol{E})$. Numbers of AChR clusters tested: 263 in WT and $120 \mathrm{in}$ MUSK $\triangle C R D$. Data are mean \pm SEM. ${ }^{*} p<0.05$ (Mann-Whitney U test). ${ }^{* * *} p<0.001$ (Mann-Whitney U test). $N=4$ embryos per genotype. Scale bar, $50 \mu \mathrm{m}$.

of muscle twitch upon nerve stimulation was significantly reduced compared to WT mice, both upon single and tetanic stimulations (strength decrease of muscle twitch: single twitch, 59\%; T40 Hz, 54\%; T60 Hz, 47\%; T80 Hz, 44\%; T100 Hz, 42\%; Fig. $7 G)$. The developed muscle specific force, defined as the muscle strength $(\mathrm{mN})$ normalized to the muscle weight $(\mathrm{g})$ was significantly reduced in MuSKDCRD compared to WT muscles, both upon single and tetanic (T100 Hz) stimulations by $52 \%$ and $24 \%$, respectively $($ Fig. $7 \mathrm{H})$. One expected pathophysiological consequence of NMJ structural changes is fatigable muscle weakness as observed in myasthenia (Hantaï et al., 2013). We therefore evaluated the muscle fatigue strength after a train of tetanic nerve stimulations $(\mathrm{T} 60 \mathrm{~Hz})$ and found that MuSKDCRD muscles exhibited a degree of fatigue more pronounced than WT muscles (fatigability increase: $30 \%$; Fig. $7 I, J$ ). Interestingly, we also observed spontaneous twitches after one twitch induced by unique stimulation in MuSK $\triangle C R D$ mice, suggesting the presence of muscle denervation processes (Fig. 7K) (Heckmann and Ludin, 1982). To further confirm this issue, noninnervated AChR clusters could be detected in P90 $M u S K \triangle C R D$ whole-mount diaphragm preparations (Fig. $7 L$ ).

Our data demonstrate that MuSK CRD deletion compromises motor performances, affects muscle strength, and leads to increased muscle fatigability. This is in agreement with clinical symptoms generally observed in patients suffering from CMS.

\section{Lithium chloride rescues NMJ phenotype of MuSKDCRD mice}

Deletion of MuSK CRD, impairing Wnt/MuSK interaction, is likely to perturb Wnt signaling at the NMJ. Indeed, treatment of WT primary myotubes with Wnt11, a member of the Wnt family known to interact with MuSK CRD and required for AChR clustering (Jing et al., 2009; Zhang et al., 2012), induced a 4.5-fold increase in the number of AChR clusters that was fully abolished ( $80 \%$ decrease) in Wnt11-treated MuSK $\triangle C R D$ primary myotubes, demonstrating that deletion of MuSK CRD alters Wnt-induced AChR clustering (Fig. $8 A$ ). In addition, $\beta$-catenin translocation to the nucleus was strongly reduced in Wnt11-treated MuSK $\triangle C R D$ compared with Wnt11treated WT primary myotubes, indicating that Wnt activation of the canonical pathway is affected in MuSKDCRD muscle cells (Fig. 8C). Because previous reports suggest that the Wnt canonical pathway is involved in neuromuscular synapse formation (Li et al., 2008; Liu et al., 2012; Wu et al., 2012a), we then reasoned that forced activation of the Wnt $\beta$-catenin signaling pathway during development could thwart impaired NMJ formation and compensate at least partially $M u S K \triangle C R D$ NMJ phenotype. To test this hypothesis, we set up a pharmacological approach using lithium chloride ( $\mathrm{LiCl})$, a wellknown reversible inhibitor of Gsk3 kinase and activator of Wnt/ $\beta$ catenin signaling (Klein and Melton, 1996; Stambolic et al., 1996; Wada, 2009). LiCl treatment of MuSK $\Delta C R D$ primary myotubes resulted in a sixfold increase in the number of AChR clusters and increased $\beta$-catenin translocation to the nucleus compared with Wnt11-treated MuSK $\triangle C R D$ myotubes, suggesting that $\mathrm{LiCl}$ is able to rescue $\mathrm{AChR}$ clustering and Wnt canonical signaling in MuSK $\triangle C R D$ myotubes (Fig. $8 B, C$ ). We then tested the effect of $\mathrm{LiCl}$ treatment on NMJ formation in vivo in MuSKDCRD mice. Repeated intraperitoneal injections of $\mathrm{LiCl}$ or placebo $(\mathrm{NaCl})$ from $\mathrm{E} 12$ to E18.5 in pregnant mice were performed, and the phenotype of E18.5 LiCl-treated MuSK $\triangle C R D$ NMJs was compared with NaCl-treated MuSK $\triangle C R D$ and WT NMJs (Fig. 8D). Remarkably, LiCl treatment almost fully rescued the postsynaptic phenotype in E18.5 MuSK $\triangle C R D$ embryos (Fig. 8D). The endplate band width in LiCltreated MuSK $\triangle C R D$ embryos was reduced by $23 \%$ compared to $\mathrm{NaCl}$-treated mutant embryos and was similar to the endplate band of WT embryos (Fig. 8E). Moreover, LiCl-treated MuSK $\triangle C R D$ embryos significantly gained AChR clusters in number (+103\%), volume $(+186 \%)$, and intensity (by 20\%) and were almost indistinguishable from WT embryos (Fig. $8 F-H$ ). In addition, presynaptic defects were improved in LiCl-treated MuSK $\Delta C R D$. The increased length of primary and secondary branches observed in $M u S K \triangle C R D$ was reduced by $35 \%$ and $28 \%$, respectively, in $\mathrm{LiCl}-$ treated MuSK $\triangle C R D$ embryos being almost similar to WT embryos (Fig. $8 I, J$ ). In addition, the number and length of bypassing neurites were reduced by $33 \%$ and $138 \%$, respectively, in LiCl-treated MuSK $\triangle C R D$ embryos compared to NaCl-treated ones (Fig. $8 K, L$ ). Together, these results indicate that $\mathrm{LiCl}$ treatment almost fully rescued both presynaptic and postsynaptic defects of MuSK $\Delta C R D$ mu- 
A WT
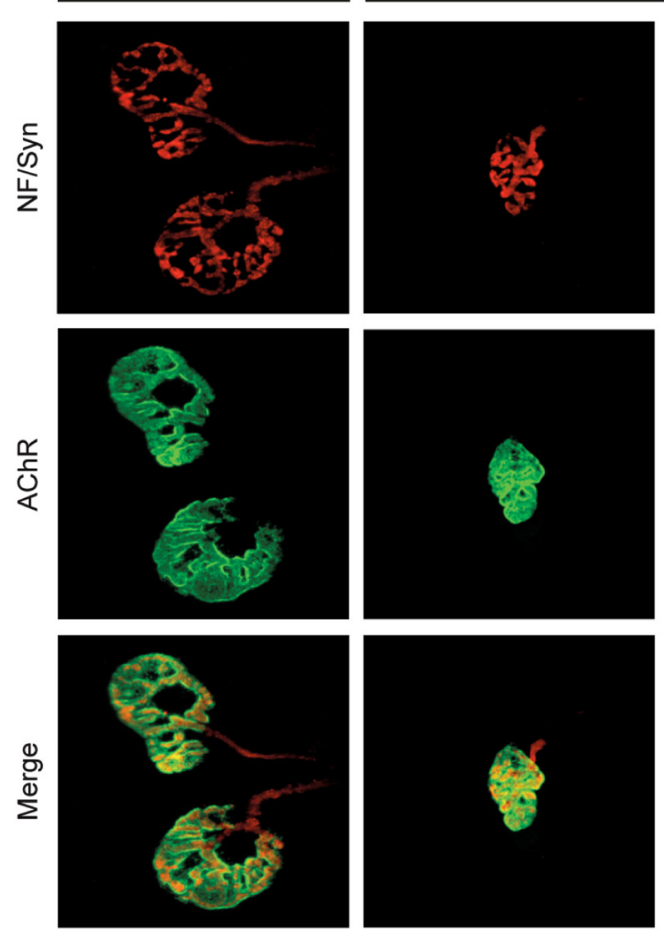

P20
MuSK $\triangle C R D$
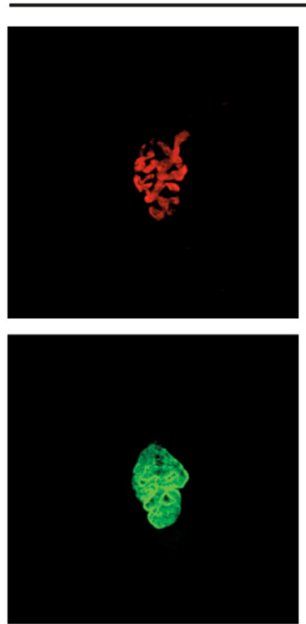
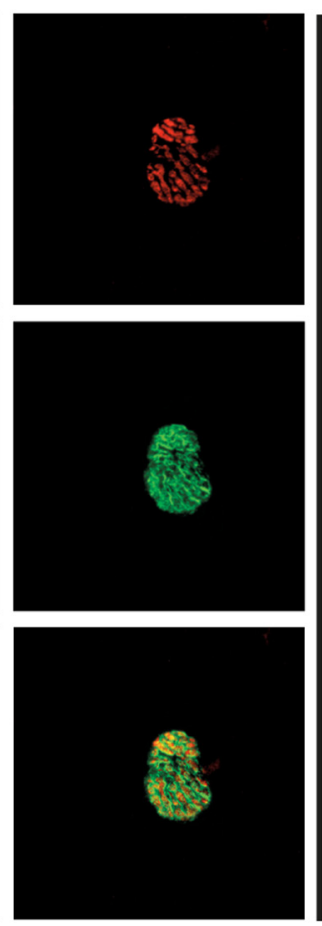

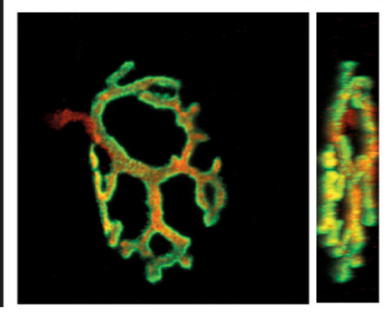

WT
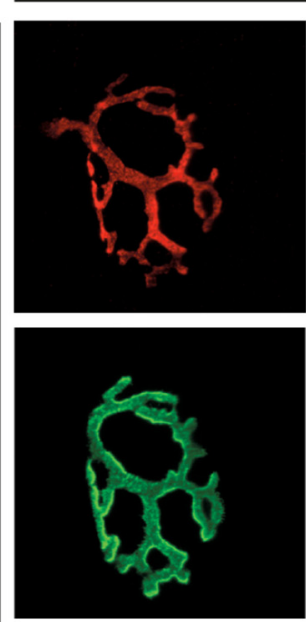

MuSK $\triangle C R D$
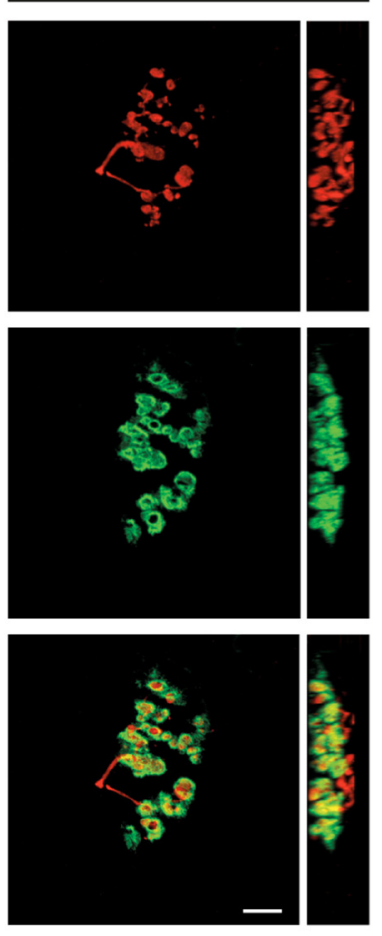

P60
B

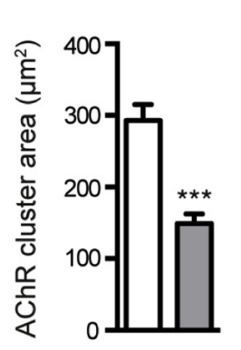

C

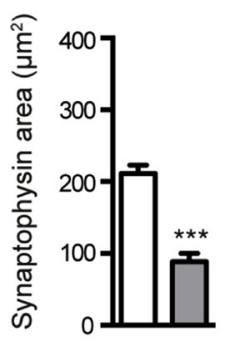

D

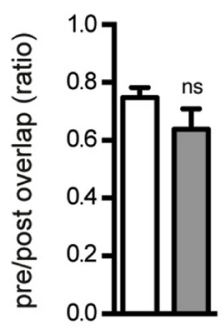

WT

MuSK $\triangle$ CRD

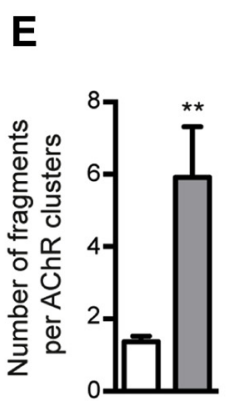

F

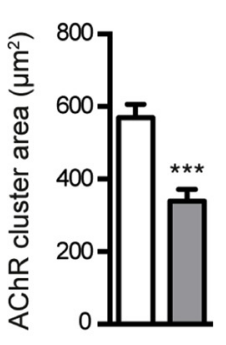

$\square \mathrm{WT}$
G

H

P20
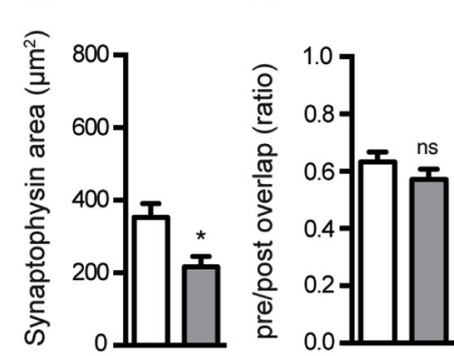

Figure 5. Immature and fragmented NMJs in MuSK $\triangle C R D$ adult mice. Whole-mount isolated muscle fibers from P20 and P60 WT and MuSK $\triangle$ CRD TA were stained with NF (red) and Syn (red) antibodies together with $\alpha$-BTX (AChRs, green). $A$, Confocal images of synapses from P20 and P60 WT and MuSK $\triangle$ CRD mice. For P60 NMJs, top views of the reconstructed image are represented on the right side. $\boldsymbol{B}-\boldsymbol{D}$, Quantification analysis of the AChR cluster area $(\boldsymbol{B})$, the Syn area $(\boldsymbol{C})$, and overlap area of presynaptic and postsynaptic elements (D) in P2O WT and MuSK $C$ CRD mice. $\boldsymbol{E}-\boldsymbol{H}$, Quantification analyses of the number of fragments per AChR clusters $(\boldsymbol{E})$, the AChR cluster area $(\boldsymbol{F})$, the Syn area $(\boldsymbol{G})$, and the overlap ratio of presynaptic and postsynaptic elements $(\boldsymbol{H})$ in P60 WT and MuSK $\triangle C R D$ mice. Data are mean \pm SEM of at least 50 NMJs. ${ }^{*} p<0.05$ (Mann-Whitney $U$ test). ${ }^{* *} p<0.01$; ${ }^{* * *} p<0.001$ (Mann-Whitney $U$ test). ns, Not significant. $N=6$ animals per genotype. Scale bar (merged image), $10 \mu \mathrm{m}$.

tants, with NMJs being phenotypically indistinguishable from WT NMJs.

To further investigate the beneficial effect of $\mathrm{LiCl}$ treatment on NMJ maintenance in adulthood, MuSK $\triangle C R D$ mice were injected with $\mathrm{LiCl}$ or placebo $(\mathrm{NaCl})$ from P10 to P60 and NMJ morphology and motor functions as well as $\beta$-catenin translocation to subsynaptic nuclei were analyzed (Fig. 9). LiCl treatment resulted in a strong increase of $\beta$-catenin translocation to subsynaptic nuclei in LiCl-treated MuSK $\triangle C R D$ compared with $\mathrm{NaCl}$ treated MuSK $\triangle C R D$ isolated P40 TA muscle fibers, as shown in the intensity plot profiles measuring the fluorescence intensity of $\beta$-catenin and DAPI along the segmented lines (Fig. 9A). In ad- dition, we found that NMJ structures of P40 TA-isolated muscle fibers from LiCl-treated $M u S K \triangle C R D$ were increased in size compared with NaCl-treated MuSK $\Delta C R D$ NMJ (Fig. 9B). The AChR cluster and Syn area in LiCl-treated mutants were increased by $40 \%$ and $105 \%$, respectively, compared to $\mathrm{NaCl}$-treated mice (Fig. 9C,D). Importantly, the number of AChRs fragments per $\mathrm{NMJ}$ in LiCl-treated mutants was decreased by $44 \%$ compared to NaCl-treated mice (Fig. 9E). Moreover, LiCl treatment significantly improved MuSK $\triangle C R D$ mice latencies to fall from the rail grip as well as fore and hindlimb strength compared to NaCltreated $M u S K \Delta C R D$ mice (Fig. $9 F-H$ ). Together, these data demonstrate that $\mathrm{LiCl}$ treatment to postnatal MuSKDCRD mice 

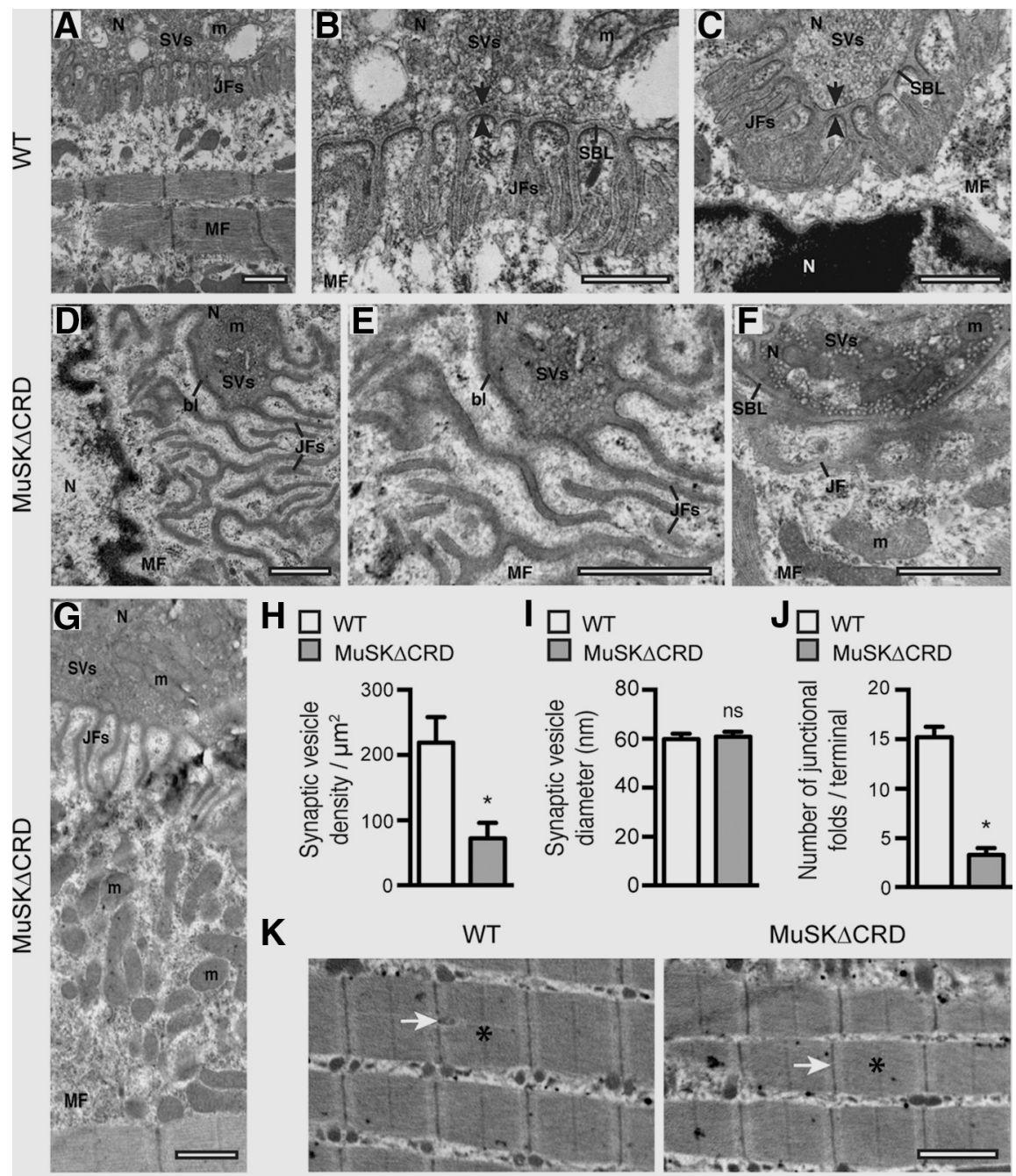

$\mathbf{K}$
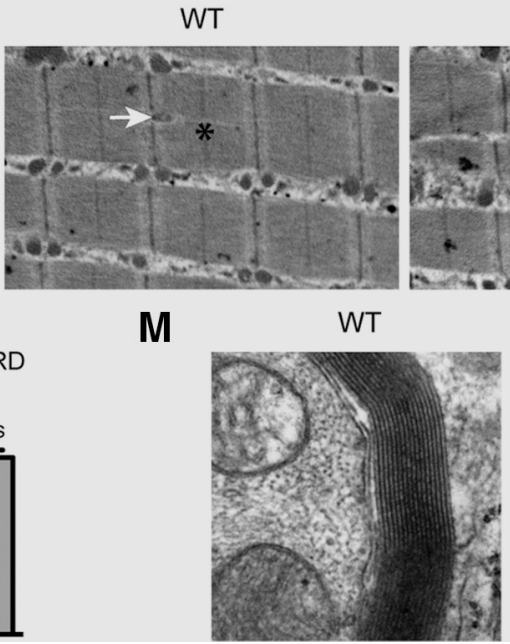

MuSK $\triangle$ CRD

$\mathbf{L}$

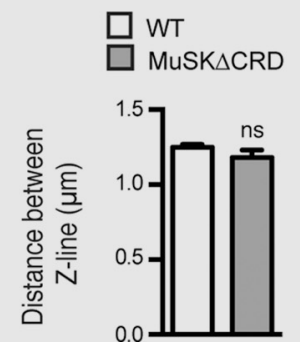

M
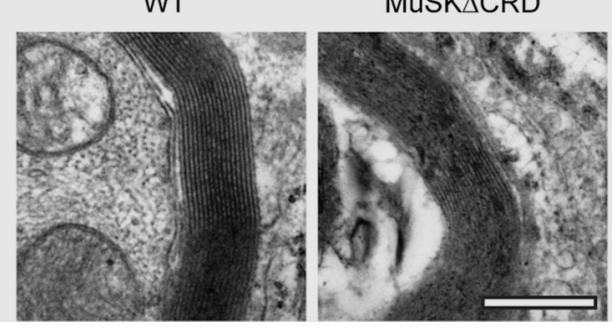

Figure 6. Disorganized NMJ ultrastructures in MuSK $\triangle C R D$ mice. $A-G$, Representative electron micrographs of $P 120$ WT and MuSK $\triangle C R D$ TA NMJs. $\boldsymbol{A}-\boldsymbol{C}$, Examples of WT NMJs. D-G, Examples of MuSK $\triangle$ CRD NMJs. $\boldsymbol{B}, \boldsymbol{E}$, Higher-magnification views of $\boldsymbol{A}$ and $\boldsymbol{D}$, respectively. $\boldsymbol{H}-\boldsymbol{J}$, Quantification analyses of the synaptic vesicle density $(\boldsymbol{H})$, diameter $(\boldsymbol{I})$, and the number of JFs $(\boldsymbol{J})$ in MuSK $\Delta C R D$ compared to WT mice. $K$, Representative electron micrograph of P120 WT and MuSK $\triangle C R D$ TA structure. $L$, Quantification of the distance between Z-lines in MuSK $\triangle C R D$ and WT mice. $\boldsymbol{M}$, Representative electron micrograph of myelin sheath in P120 WT and MuSK $\triangle$ CRD TA. Data are mean \pm SEM. ${ }^{*} p<0.05$ (Mann-Whitney $U$ test). $N=4$ animals per genotype. ns, Not significant; N, nerve; MF, muscle fiber; SVs, synaptic vesicles; $m$, mitochondria; SBL, synaptic basal lamina. Black arrows indicate presynaptic membrane. Black arrowheads indicate postsynaptic membrane. White arrows indicate Z-line. Stars indicate M-line. Scale bars: $\boldsymbol{A}-\mathbf{G}, 500 \mathrm{~nm} ; \boldsymbol{H}, \boldsymbol{J}, 1 \boldsymbol{\mu \mathrm { m }}$.

improves the NMJ morphological defects and muscle strength and restores $\beta$-catenin translocation to synaptic nuclei, suggesting that MuSK CRD plays a role during NMJ maintenance in adulthood likely in part via activation of the Wnt $\beta$-catenin signaling pathway.

\section{Discussion}

Here, we have investigated the functional role of the MuSK-Wnt binding domain (CRD) during NMJ formation and maintenance

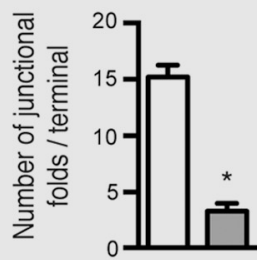

in vivo. To this end, we generated mutant mice deficient for MuSK CRD. Deletion of MuSK CRD leads to severe alteration of both presynaptic and postsynaptic elements during early muscle prepatterning (E14) and NMJ differentiation (E18.5) mainly characterized by the following: (1) a drastic deficit in AChR clusters and (2) exuberant axonal growth bypassing AChR clusters. Moreover, MuSK CRD deletion is pathogenic in adult mice, inducing CMS-like symptoms, including kyphosis, NMJ dismantlement, muscle weakness, and fatigability as previously observed in other mice models of CMS (Gomez et al., 1997; Chevessier et al., 2008, 2012; Bogdanik and Burgess, 2011; Webster et al., 2013). We also report that NMJ innervation defects in MuSK $\triangle C R D$ mice can be rescued in vivo by $\mathrm{LiCl}$ treatment. Together, our data uncover a critical role for MuSK CRD in NMJ formation and function in adulthood.

Wnts proteins are known to be involved in muscle prepatterning early during NMJ formation (Wu et al., 2010). Moreover, in zebrafish, Wnt11r induced aneural AChRs clustering requires the CRD of Unpplugged/MuSK (Jing et al., 2009). However, zebrafish lacking muscle prepatterning are able to form NMJ and are fully motile, leaving open the question of the exact role of the prepatterning in NMJ functioning (Jing et al., 2009; Gordon et al., 2012). Here, we demonstrate that deletion of MuSK CRD in mammals severely impairs muscle prepatterning because the number of AChR clusters is drastically reduced (63\%) and noninnervated $\mathrm{AChR}$ clusters are strongly increased (30\%) in E14 MuSKACRD embryos. Why deletion of MuSK CRD does not fully abolish muscle prepatterning remains unclear. Three hypotheses could explain this observation: (1) It has been shown in vitro that deletion of MuSK CRD reduces but does not fully inhibit the binding activity of Wnt proteins to MuSK (Zhang et al., 2012; Barik et al., 2014). Thus, we cannot exclude that Wnts elicited muscle prepatterning requires other domains in MuSK. (2) Frizzled (Fzd) receptors are expressed in skeletal muscle cells and could mediate Wnt signaling to contribute to muscle prepatterning (Strochlic et al., 2012; Avilés et al., 2014). (3) It has been suggested that MuSK and Lrp4 expression in early fused myofibers is sufficient to auto-activate MuSK and initiate muscle prepatterning (Kim and Burden, 2008; Burden et al., 2013). Wnts binding to MuSK CRD could therefore maintain or reinforce MuSK activation to amplify muscle prepatterning.

Our results demonstrate that MuSK $\Delta$ CRD expression at the $\mathrm{NMJ}$ is increased by $70 \%$ in mutant compared with WT mice. In 
A

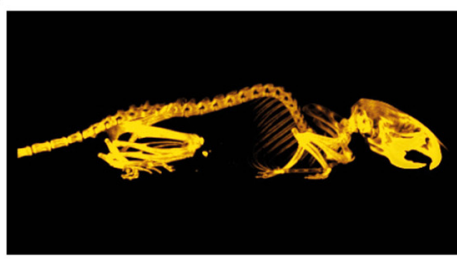

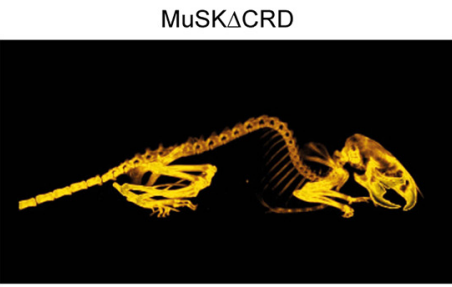

B

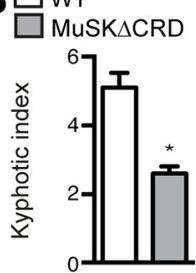

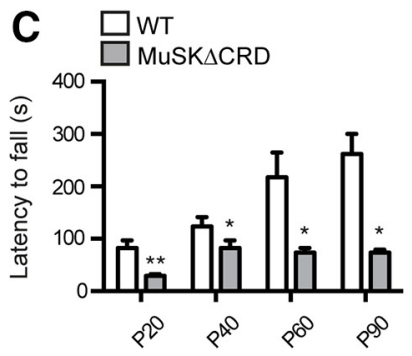

F
5
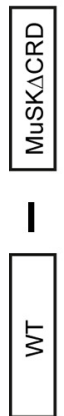

\begin{tabular}{|l|}
\hline 0 \\
$\frac{1}{0}$ \\
y \\
$\frac{1}{c}$ \\
$\stackrel{5}{\Sigma}$ \\
\hline
\end{tabular}
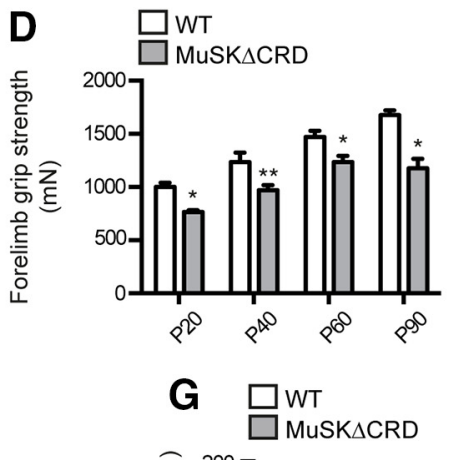
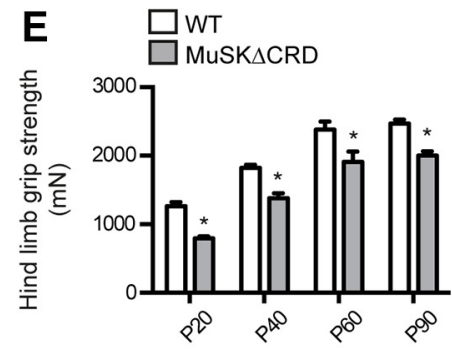

H $\square$ WT
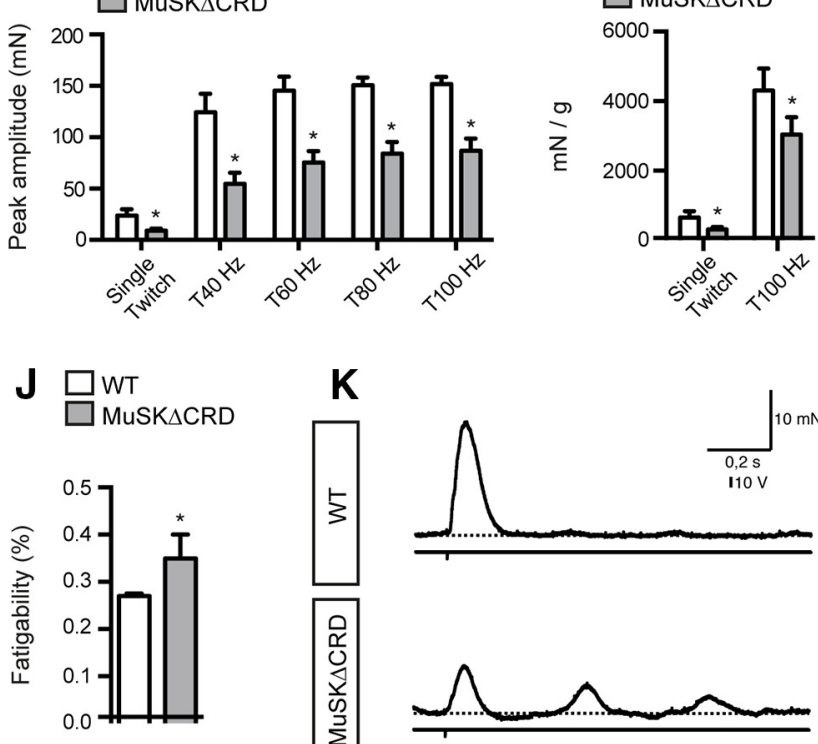

K
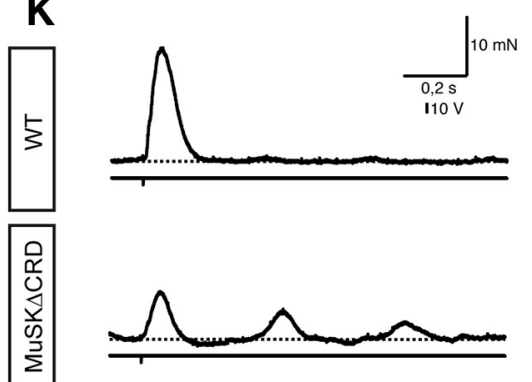

$\mathbf{L}$

WT

MUSK $\triangle$ CRD
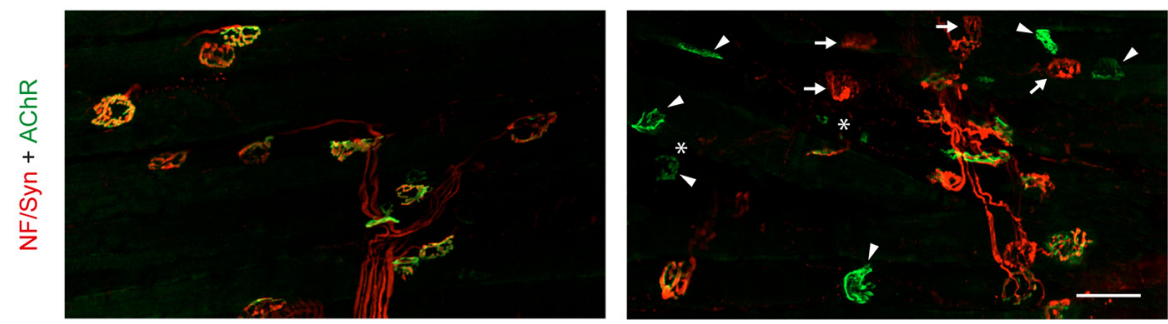

Figure 7. MuSK $\triangle C R D$ mice progressively develop muscle weakness, fatigability, and decreased muscle contraction. $A$, Micro-CT scans of P90 WT and MuSK $\triangle$ CRD mice. $B$, P120 WT and MuSK $\triangle C R D$ KI. C, Latency to fall quantifications during a rail-grip test at various time points (P20, P40, P60, and P90). D, E, Quantification of forelimb $(\boldsymbol{D})$ and hindlimb $(\boldsymbol{E})$ grip strengths in WT and MuSK $\triangle C R D$ mice. $\boldsymbol{F}$, Representative examples of twitch and tetanic contractions evoked by stimulation of the motor nerve in WT and MuSK $C R D$ P120 isolated mouse hemidiaphragms. The phrenic nerve was stimulated either with single or tetanic stimuli ( 600 ms duration) at 20,40,60,80, and $100 \mathrm{~Hz}$. $\mathbf{G}, \boldsymbol{H}$, Peak amplitudes of nerve-evoked single twitch and tetanic stimulations in WT and MuSK $\triangle C R D$ mice. $I$, Example of repeated tetanic nerve stimulation $(60 \mathrm{~Hz}, 600 \mathrm{~ms}$ duration at $1 \mathrm{~Hz}$ ) that induced a degree of fatigue more pronounced in MuSK $\triangle$ CRD than in WT.J, Quantification of the fatigability in WT and MUSK $\triangle$ CRD. $K$, Example of spontaneous twitch induced by unique stimulation observed in MuSK $\Delta C R D$ muscles. Bottom line indicates the stimulator. Calibration scales in WT apply to MuSK $\triangle C R D$. L, Confocal images of whole-mount P90 WT and MuSK $\triangle C R D$ left hemidiaphragms stained as in Figure $2 B$. White arrows indicates loss of postsynapse. White arrowheads indicates denervated postsynapses. White stars indicate fragmented NMJ. Data are mean $\pm S E M .{ }^{*} p<0.05$ (Mann-Whitney $U$ test). ${ }^{* *} p<0.01$ (Mann-Whitney $U$ test). $N=3$ animals per genotype in $\boldsymbol{A}, \boldsymbol{B}, \boldsymbol{L} . \boldsymbol{N}=6$ animals per genotype in $\boldsymbol{C}-\boldsymbol{E} . N=5$ animals per genotype in $\boldsymbol{F}-\boldsymbol{K}$. Scale bar: $L, 50 \mu \mathrm{m}$. 

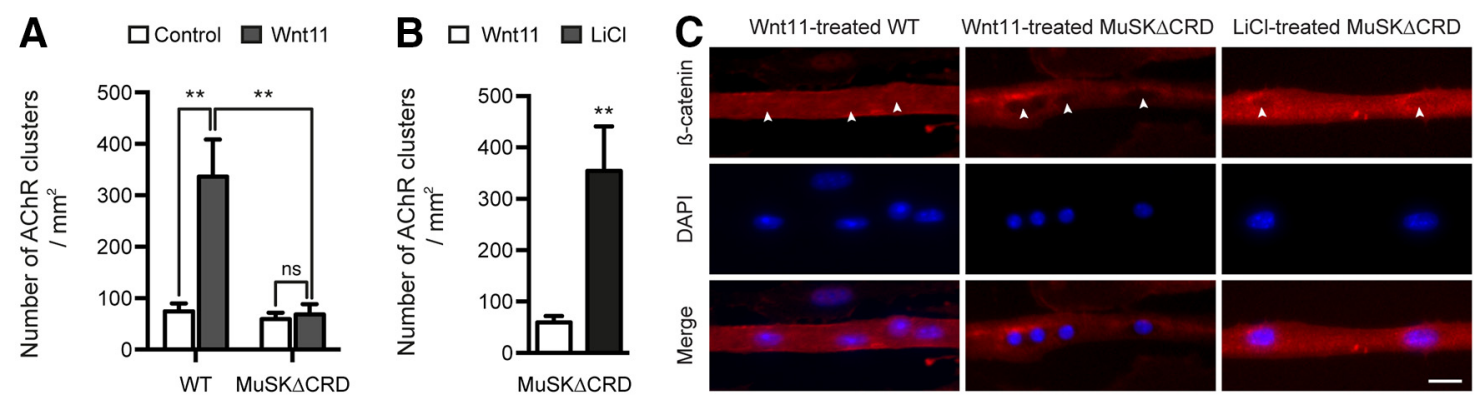

D

WT

$\mathrm{NaCl}$-treated MuSK $\triangle \mathrm{CRD}$

LiCl-treated MuSK $\triangle \mathrm{CRD}$
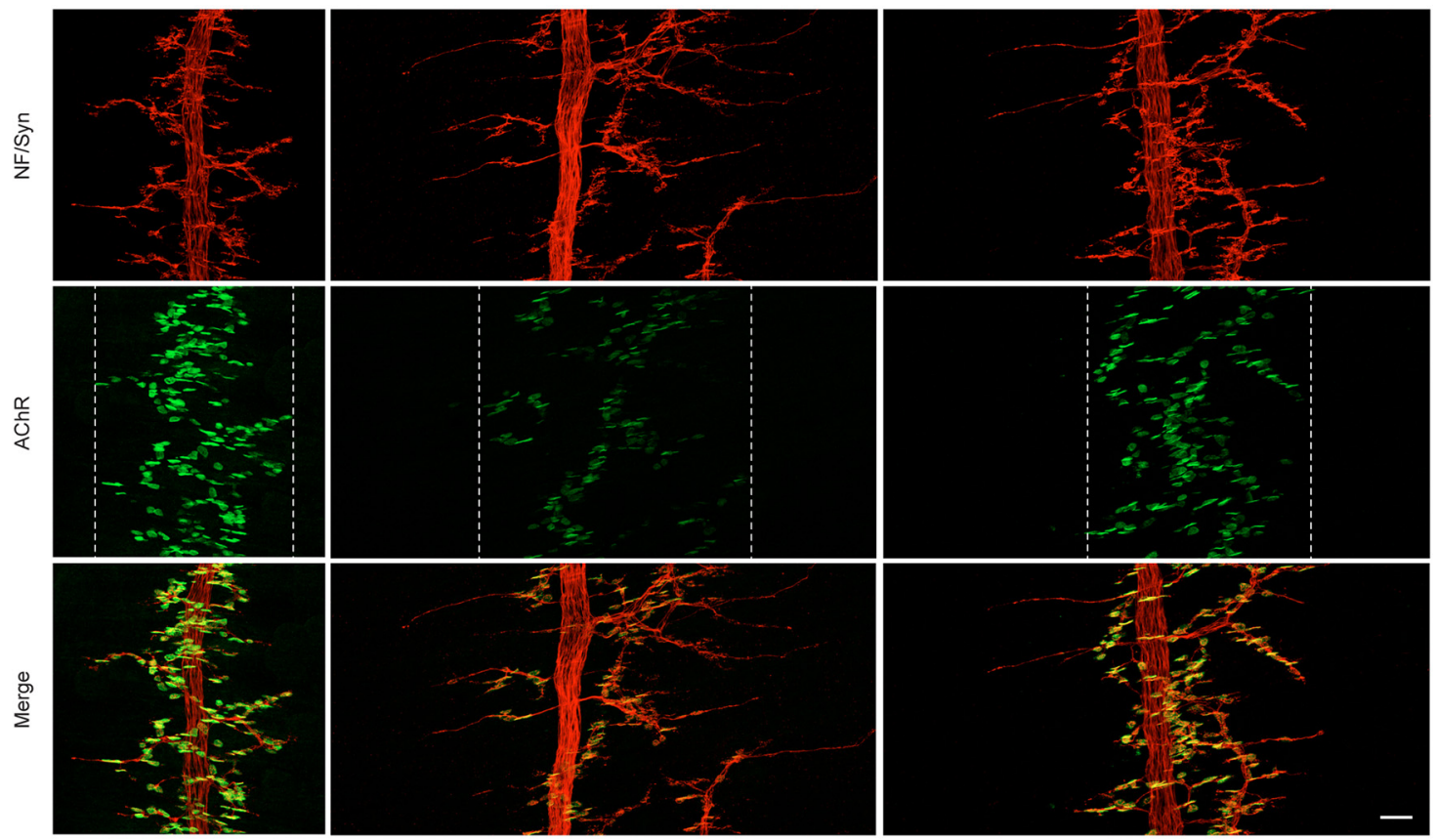

E

F

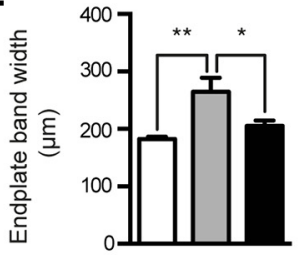

I

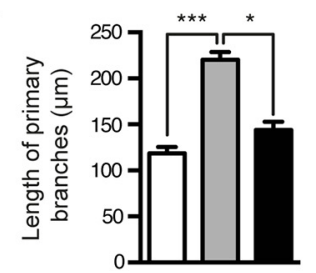

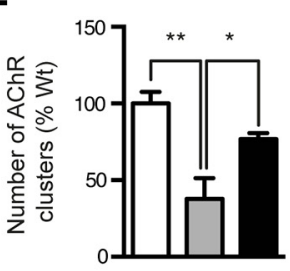

Wt

J

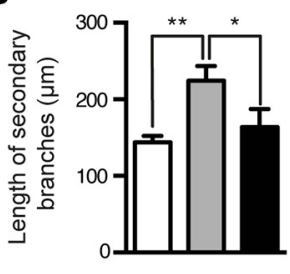

Wt
G

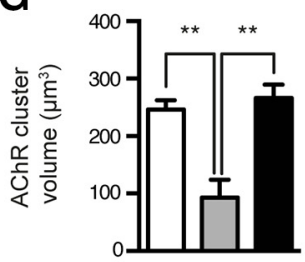

H

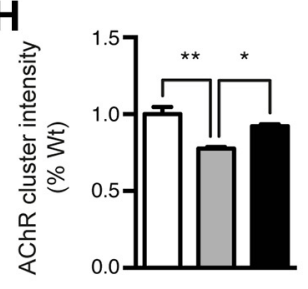

LiCl-treated MuSK $\triangle$ CRD

$\mathrm{K}$

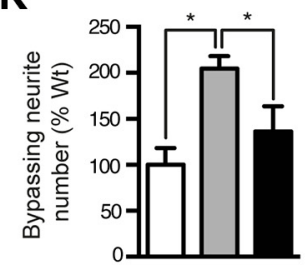

L

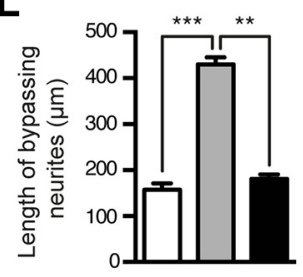

Figure 8. LiCl treatment rescues NMJ defects in MuSK $\triangle C R D$ embryos. $A, B$, Quantitative analysis of the number of AChR clusters in myotubes isolated from WT or MuSK $\triangle C R D$ primary cultures and treated or not with Wnt11 ( $\boldsymbol{A}$ ) or LiCl (B).C, Examples of Wnt11-treated WT, Wntt11-treated MuSK $\triangle$ CRD, and LiCl-treated MuSK $\Delta$ CRD primary myotubes stained with $\beta$-catenin together with DAPI to visualize $\beta$-catenin translocation to nuclei (white arrowheads). D. Confocal images of whole-mount E18.5 WT, NaCl-treated MuSK $\triangle$ CRD, and LiCl-treated MuSK $\Delta$ CRD left hemidiaphragms stained as in Figure $2 B$. White dashed lines indicate the synaptic endplate band and include most AChR clusters. $\boldsymbol{E}-\boldsymbol{L}$, Quantification of the endplate band width $(\boldsymbol{E}), A C h R$ cluster number $(\boldsymbol{F})$, volume

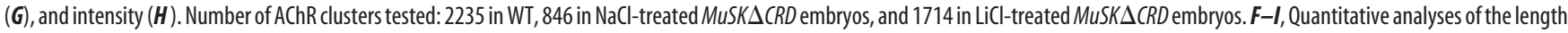
of primary and secondary nerve branches $(I, J)$, the number and the length of bypassing neurites $(\boldsymbol{K}, \boldsymbol{L})$. At least $300-400$ primary and secondary nerve branches were analyzed per condition. Data are mean \pm SEM. ${ }^{*} p<0.05$ (two-way ANOVA or Mann-Whitney $U$ test). ${ }^{* *} p<0.01$ (two-way ANOVA or Mann-Whitney $U$ test). ${ }^{* * *} p<0.001$ (two-way ANOVA or Mann-Whitney $U$ test). ns, Not significant. $N=6$ embryos per genotype. Scale bar: $\boldsymbol{C}$ (merged image), $20 \mu \mathrm{m} ; \boldsymbol{D}, 50 \mu \mathrm{m}$. 
A
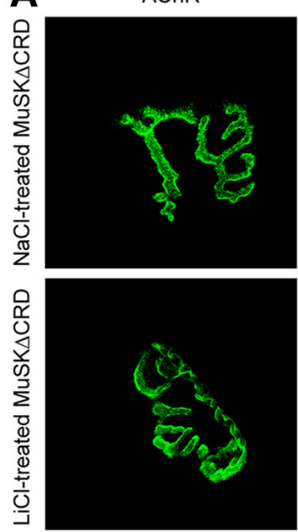

B
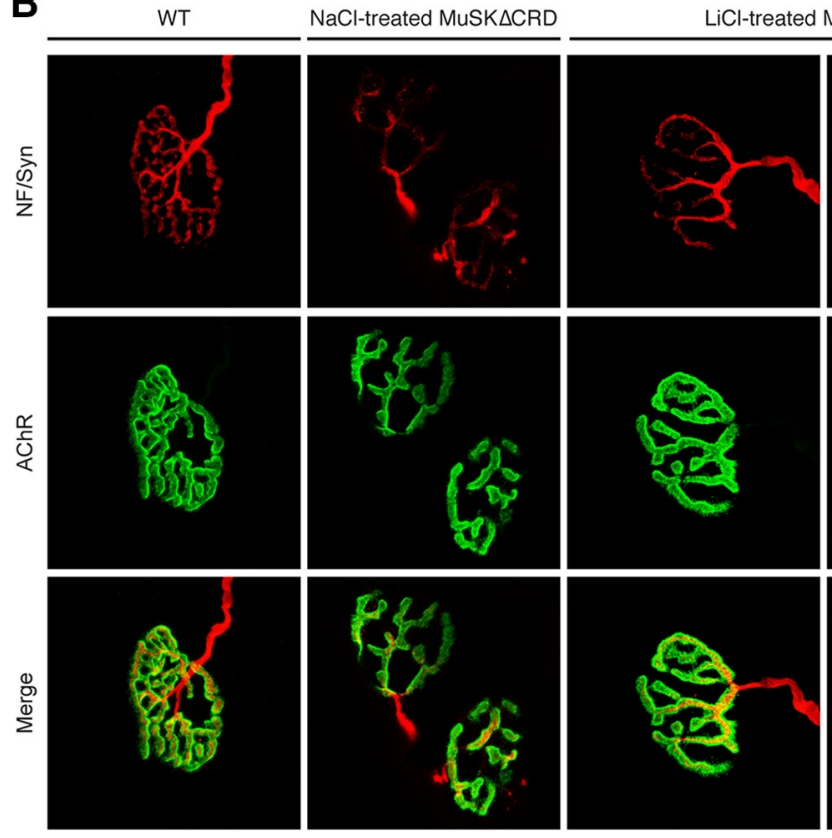

P40

$\mathbf{F}$

F

$\square \mathrm{Wt}$
$\square \mathrm{NaCl}$-treated MuSK $\triangle \mathrm{CRD}$
LiCl-treated MuSK $\triangle \mathrm{CRD}$
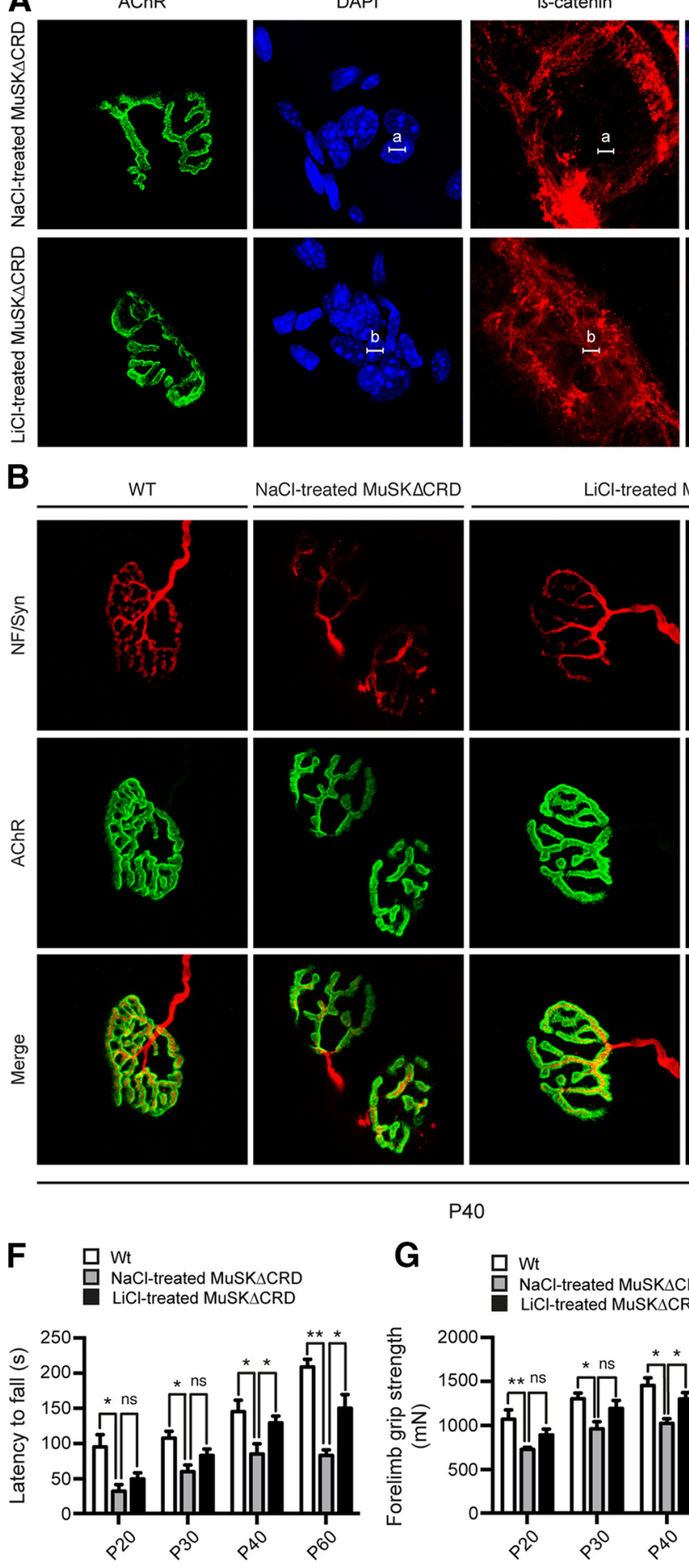

G

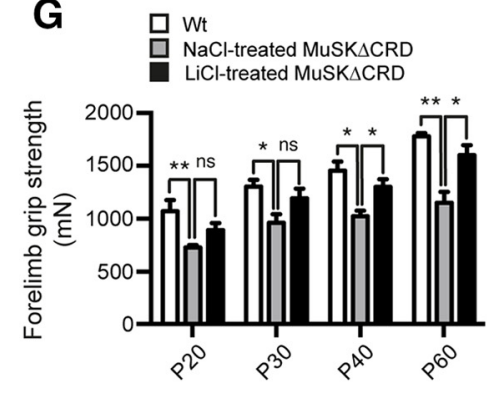

Merge

LiCl-treated MuSK $\triangle$ CRD

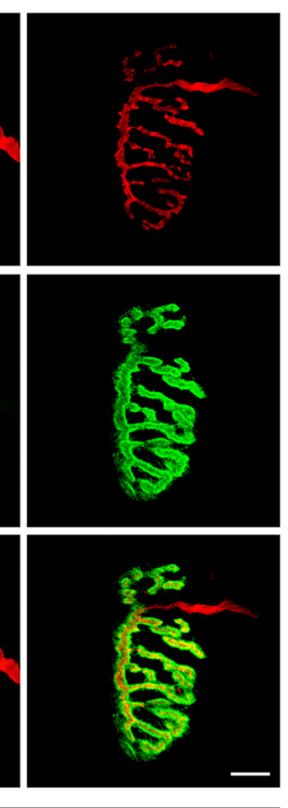

H

Q $w_{t}$

$\square \mathrm{NaCl}$-treated MuSK $\triangle \mathrm{CRD}$ LiCl-treated MuSK $\triangle \mathrm{CRD}$

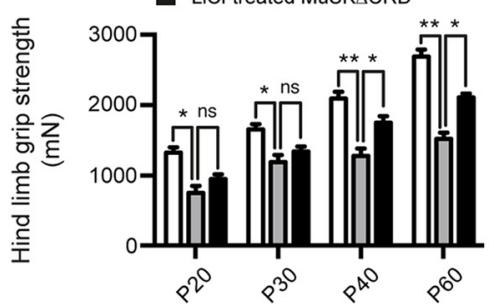

Figure 9. LiCl treatment restores NMJ morphological defects and motor function in adult MuSK $\triangle C R D$ mice. A, Confocal images of synapses from P40 NaCl-treated or LiCl-treated MuSK $\Delta C R D$ whole-mount isolated TA muscle fibers stained with $\beta$-catenin (red) antibody together with $\alpha$-BTX (AChRs, green) and DAPI (blue). Examples of intensity plot profiles measuring the fluorescence

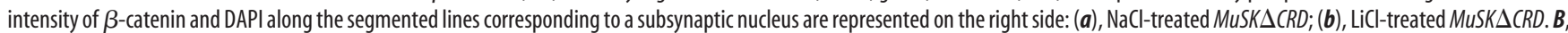
Confocal images of synapses from P40 WT, NaCl-treated, or LiCl-treated MuSK $\Delta$ CRD whole-mount isolated TA muscle fibers stained with NF (red) and Syn (red) antibodies together with $\alpha$-BTX (AChRs, green). $\boldsymbol{C}-\boldsymbol{E}$, Quantification analyses of the $A C h R$ cluster area $(\boldsymbol{C})$, the Syn area $(\boldsymbol{D})$, and the number of fragments per $A C h R$ clusters $(\boldsymbol{E})$. $\boldsymbol{F}$, Latency to fall quantifications during a rail-grip test at various time points (P20, P40, P60, and P90). G, H, Quantification of fore $(\boldsymbol{G})$ and hindlimb $(\boldsymbol{H})$ grip strength in WT, NaCl-treated, or LiCl-treated MuSKACRD mice. Data are mean \pm SEM of at least 50 NMJs. ${ }^{*} p<0.05$ (two-way ANOVA). ${ }^{* *} p<0.01$ (two-way ANOVA). ${ }^{* * *} p<0.001$ (two-way ANOVA). ns, Not significant. $N=6$ animals per genotype. Scale bar: $A, B$ (merged image), $10 \mu$ m.

zebrafish, Wnt proteins have been shown to regulate the level of MuSK expression at the plasma membrane via activation of Wntinduced MuSK endocytosis (Gordon et al., 2012). Therefore, deletion of MuSK CRD could disturb MuSK translocation from the plasma membrane to intracellular compartment, leading to MuSK membrane accumulation and reduction of Wnt/MuSK downstream signaling. Accordingly, we show that Wnt11induced AChR clustering as well as $\beta$-catenin translocation to the 
nucleus are impaired in MuSK $\triangle C R D$ primary myotubes, suggesting that deletion of MuSK CRD alters downstream Wnt canonical signaling during NMJ formation.

With innervation, nerve terminals release agrin that binds to Lrp4 and subsequently increases MuSK phosphorylation further enhancing AChR clustering (Kim et al., 2008; Zhang et al., 2008, 2011). Among the key molecules involved in NMJ formation, MuSK, Lrp4, Rapsyn, and Dok7 knock-out mice lack both aneural and agrin-induced AChR clusters (DeChiara et al., 1996; Gautam et al., 1999; Okada et al., 2006; Weatherbee et al., 2006). In contrast, although muscle prepatterning is severely affected in MuSK $\triangle C R D$ embryos, our results show that, at E18.5, upon innervation, NMJs are able to form but are abnormal with reduced AChR cluster number (30\%), volume, and density, suggesting that (1) prepatterning is not indispensable but necessary for normal progression of NMJ differentiation, and (2) innervation only partially compensates early AChR clusters deficit. Because we show that MuSK CRD deletion does not affect agrininduced AChR clustering in primary myotubes, AChR clusters defects observed are likely to result from early developmental defects. However, Wnt proteins may bind to other receptors at the postsynaptic membrane, including Fzd, thus activating signaling mechanisms leading to inhibition of agrin-induced AChR clustering. Consistent with this, it has been recently shown that Fzd9 is highly expressed in skeletal muscle when NMJs form and its overexpression in culture myotubes impairs agrin-induced AChR clustering (Avilés et al., 2014).

In MuSK and LRP4 null mutant mice, motor axons grow extensively throughout the muscle (DeChiara et al., 1996, Weatherbee et al., 2006). Similarly, both during muscle prepatterning and later during NMJ formation, MuSK $\triangle C R D$ motor axons overshoot AChR clusters and grow aberrantly all over the muscle. However, in contrast to MuSKSCRD embyros, MuSK and LRP4 null mutant exhibited increased nerve branching/arborization. These results underline the importance of the MuSK CRD in regulating a muscle retrograde stop signal for motor axons although not required for presynaptic nerve arborization. In support to this hypothesis, studies of conditional invalidation or overexpression of muscle key components of Wnt canonical signaling, including Lrp4 and $\beta$-catenin in vivo in mice, suggest a role for Wnt canonical signaling to direct a retrograde signaling required for presynaptic differentiation (Li et al., 2008; Liu et al., 2012; Wu et al., 2012a, 2012b).

Intriguingly, despite severe NMJ formation defects, MuSK $\triangle C R D$ mice are viable at birth and reach adulthood without any obvious abnormal phenotype during the first 2 weeks. In contrast, mice deficient for MuSK fail to form NMJs and die at birth due to respiratory failure (DeChiara et al., 1996). These data suggest that the remaining activity of MuSK deleted from its CRD is sufficient to prevent mutant mice from lethality. However, 2 weeks after birth, $M u S K \triangle C R D$ mice start to develop CMS-like symptoms. Morphological analysis of adult MuSK $\triangle C R D$ NMJs reveals abnormal endplates architecture, with an immature phenotype at P20 followed by a severe dismantlement at P60. This has been observed in all muscles analyzed, including diaphragm, TA, soleus, and extensor digitorum longus, suggesting that MuSK CRD is necessary for all striated muscles to guarantee NMJ integrity. This abnormal NMJ phenotype is likely to be the consequence of early NMJ defects during development because NMJ defects similar to those observed in mutant embryos are detected in P5 MuSK $\triangle C R D$ diaphragms. In this hypothesis, in addition to its role for synapse positioning early during NMJ formation, muscle prepatterning would also have an unsuspected role in NMJ func- tioning in adult mice. However, we cannot exclude a specific MuSK CRD-dependent role of Wnts during NMJ maintenance in adulthood. Further investigations are required to discriminate the role of MuSK CRD during NMJ formation in embryos and maintenance in adulthood.

NMJ fragmentation is often associated with muscle weakness as it has been described in CMS patients (Slater et al., 2006). Indeed, MuSK $\triangle C R D$ adult mice develop fatigable muscle weakness highlighted by abnormal performance in the grip test assay, ex vivo isometric diaphragm contraction, and fatigability measurement in response to nerve stimulation. Interestingly, one mutant mouse over five mice tested for isometric diaphragm contraction exhibits spontaneous twitches after a unique stimulation, a phenomenon often caused by muscle denervation (Heckmann and Ludin, 1982). In line with this observation, analysis of the NMJ innervation pattern in P90 MuSK $\triangle C R D$ mice reveals the presence of non-innervated AChR clusters suggesting a denervation-like process.

Electron microscopy analyses of adult MuSK $\triangle C R D$ NMJs reveal defects in presynaptic vesicle density and postsynaptic folds structure associated with either few or highly disorganized JFs. $\mathrm{AChR}$ clusters and JFs are required for the genesis of an efficient endplate potential leading to the activation of the voltage-gated sodium channels concentrated in the depth of the JFs (Engel and Fumagalli, 1982; Marques et al., 2000). Thus, reduced presynaptic vesicle density, fragmented endplates, and disorganized or fewer JFs are most probably responsible for the fatigable muscle weakness observed in adult MuSK $\triangle C R D$ mice. Our investigation of the muscle morphology does not reveal major changes, except mild muscle atrophy with reduced fiber size (data not shown). This muscle atrophy may participate to the muscle weakness, but it may also result from defective NMJ maintenance because muscle activity is required for muscle trophicity (Schiaffino et al., 2007).

$\mathrm{LiCl}$ is currently used as a pharmacological reagent to treat bipolar, Parkinson's, and Hungtinton's diseases (Klein and Melton, 1996; Schou, 2001; Chiu et al., 2011; Yong et al., 2011). Remarkably, $\mathrm{LiCl}$ treatment rescues $\beta$-catenin signaling and improves the impaired NMJ defects in both MuSK $\triangle C R D$ embryos and adult mice, suggesting that the defects observed during NMJ formation and maintenance in $M u S K \Delta C R D$ mice are in part due to inhibition of the Wnt canonical signaling pathway. However, given that Gsk3 is a pivotal kinase interacting with multiple signaling pathways, including PI3K-PTEN-Akt-mTOR or Ras-RafMEK-ERK, we cannot rule out the possibility that $\mathrm{LiCl}$ regulates other signaling pathways involved in NMJ development $(\mathrm{McCu}-$ brey et al., 2014). Interestingly, LiCl was shown to be efficient in the treatment of occulopharyngeal muscular dystrophy through activation of the Wnt canonical pathway and to improve skeletal muscle strength in mouse models of myotonic dystrophy (Jones et al., 2012; Abu-Baker et al., 2013). Our results provide the first evidence that $\mathrm{LiCl}$ or other $\mathrm{Gsk} 3$ inhibitors might also be used as therapeutic reagents or in complement to the treatment currently available of neuromuscular disorders associated with Wnt-MuSK deficiency.

\section{References}

Abu-Baker A, Laganiere J, Gaudet R, Rochefort D, Brais B, Neri C, Dion PA, Rouleau GA (2013) Lithium chloride attenuates cell death in oculopharyngeal muscular dystrophy by perturbing $\mathrm{Wnt} / \beta$-catenin pathway. Cell Death Dis 4:e821. CrossRef Medline

Arber S, Burden SJ, Harris AJ (2002) Patterning of skeletal muscle. Curr Opin Neurobiol 12:100-103. CrossRef Medline

Avilés EC, Pinto C, Hanna P, Ojeda J, Pérez V, De Ferrari GV, Zamorano P, 
Albistur M, Sandoval D, Henríquez JP (2014) Frizzled-9 impairs acetylcholine receptor clustering in skeletal muscle cells. Front Cell Neurosci 8:110. CrossRef Medline

Barik A, Zhang B, Sohal GS, Xiong WC, Mei L (2014) Crosstalk between Agrin and Wnt signaling pathways in development of vertebrate neuromuscular junction. Dev Neurobiol 74:828-838. CrossRef Medline

Berrih-Aknin S, Frenkian-Cuvelier M, Eymard B (2014) Diagnostic and clinical classification of autoimmune myasthenia gravis. J Autoimmun 48:143-148. CrossRef Medline

Birling MC, Dierich A, Jacquot S, Hérault Y, Pavlovic G (2012) Highlyefficient, fluorescent, locus directed cre and FlpO deleter mice on a pure C57BL/6N genetic background. Genes 50:482-489. CrossRef Medline

Bogdanik LP, Burgess RW (2011) A valid mouse model of AGRINassociated congenital myasthenic syndrome. Hum Mol Genet 20:46174633. CrossRef Medline

Bolte S, Cordelières FP (2006) A guided tour into subcellular colocalization analysis in light microscopy. J Microsc 224:213-232. CrossRef Medline

Burden SJ, Yumoto N, Zhang W (2013) The role of MuSK in synapse formation and neuromuscular disease. Cold Spring Harb Perspect Biol 5:a009167. CrossRef Medline

Cartaud A, Strochlic L, Guerra M, Blanchard B, Lambergeon M, Krejci E, Cartaud J, Legay C (2004) MuSK is required for anchoring acetylcholinesterase at the neuromuscular junction. J Cell Biol 165:505-515. CrossRef Medline

Chevessier F, Girard E, Molgó J, Bartling S, Koenig J, Hantaï D, Witzemann V (2008) A mouse model for congenital myasthenic syndrome due to MuSK mutations reveals defects in structure and function of neuromuscular junctions. Hum Mol Genet 17:3577-3595. CrossRef Medline

Chevessier F, Peter C, Mersdorf U, Girard E, Krejci E, McArdle JJ, Witzemann $\mathrm{V}$ (2012) A new mouse model for the slow-channel congenital myasthenic syndrome induced by the AChR $\varepsilon \mathrm{L} 221 \mathrm{~F}$ mutation. Neurobiol Dis 45:851-861. CrossRef Medline

Chiu CT, Liu G, Leeds P, Chuang DM (2011) Combined treatment with the mood stabilizers lithium and valproate produces multiple beneficial effects in transgenic mouse models of Huntington's disease. Neuropsychopharmacology 36:2406-2421. CrossRef Medline

DeChiara TM, Bowen DC, Valenzuela DM, Simmons MV, Poueymirou WT, Thomas S, Kinetz E, Compton DL, Rojas E, Park JS, Smith C, DiStefano PS, Glass DJ, Burden SJ, Yancopoulos GD (1996) The receptor tyrosine kinase MuSK is required for neuromuscular junction formation in vivo. Cell 85:501-512. CrossRef Medline

Engel AG, Fumagalli G (1982) Mechanisms of acetylcholine receptor loss from the neuromuscular junction. Ciba Found Symp 90:197-224. Medline

Gautam M, DeChiara TM, Glass DJ, Yancopoulos GD, Sanes JR (1999) Distinct phenotypes of mutant mice lacking agrin, MuSK, or rapsyn. Brain Res Dev Brain Res 114:171-178. CrossRef Medline

Gomez CM, Maselli R, Gundeck JE, Chao M, Day JW, Tamamizu S, Lasalde JA, McNamee M, Wollmann RL (1997) Slow-channel transgenic mice: a model of postsynaptic organellar degeneration at the neuromuscular junction. J Neurosci 17:4170-4179. Medline

Gordon LR, Gribble KD, Syrett CM, Granato M (2012) Initiation of synapse formation by Wnt-induced MuSK endocytosis. Development 139:10231033. CrossRef Medline

Hantaï D, Nicole S, Eymard B (2013) Congenital myasthenic syndromes: an update. Curr Opin Neurol 26:561-568. CrossRef Medline

Heckmann R, Ludin HP (1982) Differentiation of spontaneous activity from normal and denervated skeletal muscle. J Neurol Neurosurg Psychiatry 45:331-336. CrossRef Medline

Henriquez JP, Webb A, Bence M, Bildsoe H, Sahores M, Hughes SM, Salinas PC (2008) Wnt signaling promotes AChR aggregation at the neuromuscular synapse in collaboration with agrin. Proc Natl Acad Sci U S A 105: 18812-18817. CrossRef Medline

Jing L, Lefebvre JL, Gordon LR, Granato M (2009) Wnt signals organize synaptic prepattern and axon guidance through the zebrafish unplugged/ MuSK receptor. Neuron 61:721-733. CrossRef Medline

Jones K, Wei C, Iakova P, Bugiardini E, Schneider-Gold C, Meola G, Woodgett J, Killian J, Timchenko NA, Timchenko LT (2012) GSK3 $\beta$ mediates muscle pathology in myotonic dystrophy. J Clin Invest 122: 4461-4472. CrossRef Medline

Kim N, Burden SJ (2008) MuSK controls where motor axons grow and form synapses. Nat Neurosci 11:19-27. CrossRef Medline
Kim N, Stiegler AL, Cameron TO, Hallock PT, Gomez AM, Huang JH, Hubbard SR, Dustin ML, Burden SJ (2008) Lrp4 is a receptor for Agrin and forms a complex with MuSK. Cell 135:334-342. CrossRef Medline

Klein PS, Melton DA (1996) A molecular mechanism for the effect of lithium on development. Proc Natl Acad Sci U S A 93:8455-8459. CrossRef Medline

Kummer TT, Misgeld T, Sanes JR (2006) Assembly of the postsynaptic membrane at the neuromuscular junction: paradigm lost. Curr Opin Neurobiol 16:74-82. CrossRef Medline

Laws N, Hoey A (2004) Progression of kyphosis in mdx mice. J Appl Physiol 97:1970-1977. CrossRef Medline

Lin W, Burgess RW, Dominguez B, Pfaff SL, Sanes JR, Lee KF (2001) Distinct roles of nerve and muscle in postsynaptic differentiation of the neuromuscular synapse. Nature 410:1057-1064. CrossRef Medline

Liu Y, Sugiura Y, Wu F, Mi W, Taketo MM, Cannon S, Carroll T, Lin W (2012) $\beta$-Catenin stabilization in skeletal muscles, but not in motor neurons, leads to aberrant motor innervation of the muscle during neuromuscular development in mice. Dev Biol 366:255-267. CrossRef Medline

Li XM, Dong XP, Luo SW, Zhang B, Lee DH, Ting AK, Neiswender H, Kim CH, Carpenter-Hyland E, Gao TM, Xiong WC, Mei L (2008) Retrograde regulation of motoneuron differentiation by muscle beta-catenin. Nat Neurosci 11:262-268. CrossRef Medline

Marques MJ, Conchello JA, Lichtman JW (2000) From plaque to pretzel: fold formation and acetylcholine receptor loss at the developing neuromuscular junction. J Neurosci 20:3663-3675. Medline

McCubrey JA, Steelman LS, Bertrand FE, Davis NM, Sokolosky M, Abrams SL, Montalto G, D'Assoro AB, Libra M, Nicoletti F, Maestro R, Basecke J, Rakus D, Gizak A, Demidenko ZN, Cocco L, Martelli AM, Cervello M (2014) GSK-3 as potential target for therapeutic intervention in cancer. Oncotarget 5:2881-2911. Medline

Mihaylova V, Salih MM, Mukhtar MM, Abuzeid HA, El-Sadig SM, von der Hagen M, Huebner A, Nürnberg G, Abicht A, Müller JS, et al. (2009) Refinement of the clinical phenotype in musk-related congenital myasthenic syndromes. Neurology 73:1926-1928. CrossRef Medline

Okada K, Inoue A, Okada M, Murata Y, Kakuta S, Jigami T, Kubo S, Shiraishi H, Eguchi K, Motomura M, Akiyama T, Iwakura Y, Higuchi O, Yamanashi Y (2006) The muscle protein Dok-7 is essential for neuromuscular synaptogenesis. Science 312:1802-1805. CrossRef Medline

Punga AR, Maj M, Lin S, Meinen S, Rüegg MA (2011) MuSK levels differ between adult skeletal muscles and influence postsynaptic plasticity. Eur J Neurosci 33:890-898. CrossRef Medline

Schiaffino S, Sandri M, Murgia M (2007) Activity-dependent signaling pathways controlling muscle diversity and plasticity. J Physiol 22:269278. CrossRef Medline

Schou M (2001) Lithium treatment at 52. J Affect Disord 67:21-32. CrossRef Medline

Sigoillot SM, Bourgeois F, Lambergeon M, Strochlic L, Legay C (2010) ColQ controls postsynaptic differentiation at the neuromuscular junction. J Neurosci 30:13-23. CrossRef Medline

Slater CR, Fawcett PRW, Walls TJ, Lyons PR, Bailey SJ, Beeson D, Young C, Gardner-Medwin D (2006) Pre- and post-synaptic abnormalities associated with impaired neuromuscular transmission in a group of patients with "limb-girdle myasthenia." Brain J Neurol 129:2061-2076. CrossRef Medline

Stambolic V, Ruel L, Woodgett JR (1996) Lithium inhibits glycogen synthase kinase- 3 activity and mimics wingless signalling in intact cells. Curr Biol 6:1664-1668. CrossRef Medline

Strochlic L, Falk J, Goillot E, Sigoillot S, Bourgeois F, Delers P, Rouvière J, Swain A, Castellani V, Schaeffer L, Legay C (2012) Wnt4 participates in the formation of vertebrate neuromuscular junction. PLoS One 7:e29976. CrossRef Medline

Takamori M (2012) Structure of the neuromuscular junction: function and cooperative mechanisms in the synapse. Ann N Y Acad Sci 1274:14-23. CrossRef Medline

Takamori M, Nakamura T, Motomura M (2013) Antibodies against Wnt receptor of muscle-specific tyrosine kinase in myasthenia gravis. J Neuroimmunol 254:183-186. CrossRef Medline

Wada A (2009) Lithium and neuropsychiatric therapeutics: neuroplasticity via glycogen synthase kinase-3beta, beta-catenin, and neurotrophin cascades. J Pharmacol Sci 110:14-28. CrossRef Medline

Wang J, Ruan NJ, Qian L, Lei WL, Chen F, Luo ZG (2008) Wnt/beta-catenin signaling suppresses Rapsyn expression and inhibits acetylcholine receptor clustering at the neuromuscular junction. J Biol Chem 283:2166821675. CrossRef Medline 
Weatherbee SD, Anderson KV, Niswander LA (2006) LDL-receptor-related protein 4 is crucial for formation of the neuromuscular junction. Development 133:4993-5000. CrossRef Medline

Webster RG, Cossins J, Lashley D, Maxwell S, Liu WW, Wickens JR, MartinezMartinez P, de Baets M, Beeson D (2013) A mouse model of the slow channel myasthenic syndrome: neuromuscular physiology and effects of ephedrine treatment. Exp Neurol 248:286-298. CrossRef Medline

Wu H, Xiong WC, Mei L (2010) To build a synapse: signaling pathways in neuromuscular junction assembly. Development 137:1017-1033. CrossRef Medline

Wu H, Lu Y, Barik A, Joseph A, Taketo MM, Xiong WC, Mei L (2012a) $\beta$-Catenin gain of function in muscles impairs neuromuscular junction formation. Development 139:2392-2404. CrossRef Medline

Wu H, Lu Y, Shen C, Patel N, Gan L, Xiong WC, Mei L (2012b) Distinct roles of muscle and motoneuron LRP4 in neuromuscular junction formation. Neuron 75:94-107. CrossRef Medline
Yang X, Arber S, William C, Li L, Tanabe Y, Jessell TM, Birchmeier C, Burden SJ (2001) Patterning of muscle acetylcholine receptor gene expression in the absence of motor innervation. Neuron 30:399-410. CrossRef Medline

Yong Y, Ding H, Fan Z, Luo J, Ke ZJ (2011) Lithium fails to protect dopaminergic neurons in the 6-OHDA model of Parkinson's disease. Neurochem Res 36:367-374. CrossRef Medline

Zhang B, Luo S, Wang Q, Suzuki T, Xiong WC, Mei L (2008) LRP4 serves as a coreceptor of agrin. Neuron 60:285-297. CrossRef Medline

Zhang B, Liang C, Bates R, Yin Y, Xiong WC, Mei L (2012) Wnt proteins regulate acetylcholine receptor clustering in muscle cells. Mol Brain 5:7. CrossRef Medline

Zhang W, Coldefy AS, Hubbard SR, Burden SJ (2011) Agrin binds to the $\mathrm{N}$-terminal region of Lrp4 protein and stimulates association between Lrp4 and the first immunoglobulin-like domain in muscle-specific kinase (MuSK). J Biol Chem 286:40624-40630. CrossRef Medline 